\title{
NUMERICAL APPROXIMATION OF COROTATIONAL DUMBBELL MODELS FOR DILUTE POLYMERS
}

\author{
JOHN W. BARRETT* AND ENDRE SÜLI ${ }^{*}$
}

\begin{abstract}
We construct a general family of Galerkin methods for the numerical approximation of weak solutions to a coupled microscopic-macroscopic bead-spring model that arises from the kinetic theory of dilute solutions of polymeric liquids with noninteracting polymer chains. The model consists of the unsteady incompressible NavierStokes equations in a bounded domain $\Omega \subset \mathbb{R}^{d}, d=2$ or 3 , for the velocity and the pressure of the fluid, with an elastic extra-stress tensor as right-hand side in the momentum equation. The extra-stress tensor stems from the random movement of the polymer chains and is defined through the associated probability density function which satisfies a Fokker-Planck type parabolic equation, a crucial feature of which is the presence of a centre-of-mass diffusion term. We focus on finitely-extensible nonlinear elastic, FENE-type, dumbbell models. In the case of a corotational drag term we perform a rigorous passage to the limit as the spatial and temporal discretization parameters tend to zero, and show that a (sub)sequence of numerical solutions converges to a weak solution of this coupled Navier-Stokes-Fokker-Planck system.
\end{abstract}

Key words. Galerkin methods, polymeric flow models, existence of weak solutions, Navier-Stokes equations, Fokker-Planck equations, FENE.

AMS subject classifications. 76D03, 82C31, 82D60

1. Introduction. This paper is concerned with the construction and convergence analysis of Galerkin approximations to weak solutions of a system of nonlinear partial differential equations that arises from the kinetic theory of dilute polymer solutions. The solvent is an incompressible, viscous, isothermal Newtonian fluid confined to an open set $\Omega \subset \mathbb{R}^{d}, d=2$ or 3 , with boundary $\partial \Omega$. For the sake of simplicity of presentation we shall suppose that $\Omega$ has solid boundary $\partial \Omega$; the velocity field $\underset{\sim}{u}$ will then satisfy the no-slip boundary condition $\underset{\sim}{u}=0 \underset{\sim}{0}$ on $\partial \Omega$. The polymer chains which are suspended in the solvent are assumed not to interact with each other. The conservation of momentum and mass equations for the solvent then have the form of the incompressible NavierStokes equations in which the elastic extra-stress tensor $\underset{\approx}{\tau}$ (i.e., the polymeric part of the Cauchy stress tensor,) appears as a source term:

Find $\underset{\sim}{u}:(\underset{\sim}{x}, t) \in \mathbb{R}^{d+1} \mapsto \underset{\sim}{u}(\underset{\sim}{x}, t) \in \mathbb{R}^{d}$ and $p:(\underset{\sim}{x}, t) \in \mathbb{R}^{d+1} \mapsto p(\underset{\sim}{x}, t) \in \mathbb{R}$ such that

$$
\begin{aligned}
& \frac{\partial \widetilde{u}}{\partial t}+\left(\underset{\sim}{u} \cdot \nabla_{x}\right) \underset{\sim}{u}-\nu \Delta_{x} \underset{\sim}{u}+\nabla_{x} p=\nabla_{x} \cdot \underset{\sim}{\tau} \quad \text { in } \Omega \times(0, T], \\
& \nabla_{x} \cdot \underset{\sim}{u}=0 \quad \text { in } \Omega \times(0, T], \\
& \underset{\sim}{u}=\underset{\sim}{0} \quad \text { on } \partial \Omega \times(0, T], \\
& u(\underset{\sim}{x}, 0)={\underset{\sim}{u}}^{0}(\underset{\sim}{x}) \quad \forall \underset{\sim}{x} \in \Omega \text {; }
\end{aligned}
$$

where $u$ is the velocity field, $p$ is the pressure, and $\nu \in \mathbb{R}_{>0}$ is the viscosity of the solvent. For the sake of simplicity we shall assume that there are no body forces present: the presence of a body force $f \in L^{2}\left(\mathbb{R},\left[H^{-1}(\Omega)\right]^{d}\right)$ on the right-hand side of (1.1a) would not cause any particular technical complications. The extra stress tensor $\underset{\sim}{\tau}$ is defined as the second moment of $\psi$, the probability density function of the (random) conformation vector of the polymer molecules. As will be seen below, the Kolmogorov equation satisfied by $\psi$ is a Fokker-Planck type second-order parabolic equation whose transport coefficients depend on the velocity field $\underset{\sim}{u}$.

Polymer solutions exhibit a range of non-Newtonian flow properties: in particular, the stress endured by a fluid element depends upon the history of deformations experienced by that element.

\footnotetext{
*Dept. of Mathematics, Imperial College London, London SW7 2AZ, UK. email: j.barrett@ imperial.ac.uk

†OUCL, University of Oxford, Parks Road, Oxford OX1 3QD, UK. email: endre.suli@ comlab.ox.ac.uk
} 
Thereby, rheological properties of non-Newtonian fluids are governed by the flow-induced evolution of their internal microstructure. Following Keunings [27], a relevant feature of the microstructure is the conformation of the macromolecules, i.e., their orientation and the degree of stretching they experience. Since the macroscopic stress carried by each fluid element is governed by the distribution of polymer conformations within that element, from the macroscopic viewpoint it is only the statistical distribution of conformations that matters. Motivated by this observation, kinetic theories of polymeric fluids ignore quantum mechanical and atomistic effects, and focus on 'coarse-grained' models of the polymeric conformations. Depending on the level of coarse-graining, one may arrive at a hierarchy of kinetic models. For example, a dilute solution of linear polymers in a Newtonian solvent can be described in some detail by the freely jointed bead-rod Kramers chain, which comprises a number of beads (of the order of 100) connected by rigid linear segments. A coarser model of the same polymer is the freely jointed bead-spring chain, a Rouse chain, consisting of a smaller number of beads (of the order of 10) connected linearly by entropic springs. A coarser model still is the dumbbell model which involves two beads connected by an elastic spring [7]; the elastic force $\underset{\sim}{F}: D \subseteq \mathbb{R}^{d} \rightarrow \mathbb{R}^{d}$ of the spring connecting the two beads is defined by a (sufficiently smooth) spring potential $U: \mathbb{R}_{\geq 0} \rightarrow \mathbb{R}_{\geq 0}$ through

$$
\underset{\sim}{F}(\underset{\sim}{q})=H U^{\prime}\left(\frac{1}{2}|q|^{2}\right) \underset{\sim}{q}, \quad \underset{\sim}{q} \in D
$$

where $H \in \mathbb{R}_{>0}$ is a spring constant. The elongation (or conformation) vector $\underset{\sim}{q}$, whose direction and length define the direction and length of the polymer chain represented by the dumbbell, is assumed to be confined to a balanced convex open set $D \subset \mathbb{R}^{d}$; the term balanced means that $0 \in D$, and $-\underset{\sim}{q} \in D$ whenever $\underset{\sim}{q} \in D$. Typically, $D$ is an open $d$-dimensional ball of fixed radius $\sqrt{b}$, $b>0$, or an ellipse with fixed half-axes, or the whole of $\mathbb{R}^{d}$. Our analytical results in this paper are concerned with the physically realistic case when $D$ is bounded, although we shall also comment on the idealized situation when $D=\mathbb{R}^{d}$.

The governing equations of the dumbbell model considered here are $(1.1 \mathrm{a}-\mathrm{d})$, where the elastic extra-stress tensor $\underset{\sim}{\tau}$ is defined by the Kramers expression:

$$
\underset{\sim}{\tau}(\underset{\sim}{x}, t)=k \mu\left(\int_{D} \underset{\sim}{q} q^{\top} U^{\prime}\left(\frac{1}{2}|\underset{\sim}{q}|^{2}\right) \psi(\underset{\sim}{x}, \underset{\sim}{q}, t) \mathrm{d} \underset{\sim}{q}-\rho(\underset{\sim}{x}, t) \underset{\sim}{I}\right) ;
$$

here $k$ is the Boltzmann constant and $\mu$ is the absolute temperature. Further,

$$
\rho(\underset{\sim}{x}, t)=\int_{D} \psi(\underset{\sim}{x} \underset{\sim}{q}, t) \mathrm{d} \underset{\sim}{q},
$$

signifies density, and the probability density function $\psi(\underset{\sim}{x} \underset{\sim}{q}, t)$ is a solution to the Fokker-Planck equation

$$
\frac{\partial \psi}{\partial t}+\left(\underset{\sim}{u} \cdot \nabla_{x}\right) \psi+\nabla_{q} \cdot\left(\left({\underset{\sim}{\nabla}}_{x} \underset{\sim}{u} \underset{\sim}{q} \psi\right)=\varepsilon \Delta_{x} \psi+\frac{1}{2 \lambda} \underset{\sim}{\nabla_{q}} \cdot\left(\nabla_{q} \psi+U^{\prime} \underset{\sim}{q} \psi\right) .\right.
$$

Here $\lambda \in \mathbb{R}_{>0}$ and $\varepsilon \in \mathbb{R}_{>0}$ are fixed positive real numbers, called the relaxation time and the centreoff-mass diffusion coefficient, respectively. We refer to [3] for the derivation of the model; see also the recent paper of Schieber [42] for a justification of the presence of the $\underset{\sim}{x}$-dissipative centre-of-mass diffusion term $\varepsilon \Delta_{x} \psi$ on the right-hand side of (1.5).

In classical derivations of bead-spring models the centre-of-mass diffusion term is routinely omitted from the Fokker-Planck equation, on the grounds that it is several orders of magnitude smaller than the other terms in the equation. Indeed, Bhave, Armstrong, and Brown [6] estimate it to be typically in the range of about $10^{-9}$ to $10^{-7}$. However, as has been emphasized in [3], 
the omission of the term $\varepsilon \Delta_{x} \psi$ from (1.5) in the case of a heterogeneous solvent-velocity $\underset{\sim}{u(\underset{\sim}{x}, t)}$ is a mathematically counterproductive model-reduction. When $\varepsilon \Delta_{x} \psi$ is absent, (1.5) becomes a degenerate parabolic equation exhibiting hyperbolic behaviour with respect to $(\underset{\sim}{x}, t)$. Since the study of weak solutions to the coupled problem requires one to work with velocity fields $\underset{\sim}{u}$ that have very limited Sobolev regularity (typically $\underset{\sim}{u} \in L^{\infty}\left(0, T ; L_{\sim}^{2}(\Omega)\right) \cap L^{2}\left(0, T ; \underset{\sim}{H_{0}^{1}}(\Omega)\right)$ ), one is then forced into the technically unpleasant framework of hyperbolically degenerate parabolic equations with rough transport coefficients [1]. The resulting difficulties are further exacerbated by the fact that, when $D$ is bounded, a typical spring force $\underset{\sim}{F} \underset{\sim}{q})$ for a finitely-extensible model, such as the FENE (finitely-extensible nonlinear elastic) model for example, where

$$
U(s)=-\frac{b}{2} \ln \left(1-\frac{2 s}{b}\right), \quad s \in\left[0, \frac{b}{2}\right),
$$

explodes as $q$ approaches $\partial D$; see Section 2.2 below. Parabolic PDEs with unbounded coefficients are studied, for example, in the monographs of Cerrai [12] and Lorenzi and Bertoldi [33]. We note in passing that, on letting $b \rightarrow+\infty$, the FENE potential converges to the (linear) Hookean spring potential $U(s)=s$ while $D$ then becomes the whole of $\mathbb{R}^{d}$, - corresponding to a mathematically simple(r) albeit physically unrealistic scenario in which a polymer chain can have arbitrarily large elongation.

An early effort to show the existence and uniqueness of local-in-time solutions to a family of bead-spring type polymeric flow models is due to Renardy [41]. While the class of potentials $\underset{\sim}{F}(\underset{\sim}{q})$ considered by Renardy [41] (cf. hypotheses $(\mathrm{F})$ and $\left(\mathrm{F}^{\prime}\right)$ on pp. 314-315) does include the case of Hookean dumbbells, it excludes the practically relevant case of the FENE model (see Section 2.2 below). More recently, E, Li, and Zhang [18] and Li, Zhang, and Zhang [29] have revisited the question of local existence of solutions for dumbbell models.

Constantin [14] has considered the Navier-Stokes equations coupled to nonlinear Fokker-Planck equations describing the evolution of the probability distribution of the particles interacting with the fluid. He showed global existence of smooth solutions if inertial effects are neglected. The necessary relationship (eq. (2.14) in [14]) for the existence of a Lyapunov function in the sense of Theorem 2.2 of [14] does not hold for the polymer models considered in the present paper.

Otto and Tzavaras [38] have investigated the Doi model (which is similar to a Hookean model, except that $D=S^{2}$ ) for suspensions of rod-like molecules in the dilute regime. For certain parameter values, the velocity gradient vs. stress relation defined by the stationary and homogeneous flow is not rank-one monotone. They considered the evolution of possibly large perturbations of stationary flows and proved that, even in the absence of a microscopic cut-off, discontinuities in the velocity gradient cannot occur in finite time.

In the case of Hookean dumbbells, and assuming $\varepsilon=0$, the coupled microscopic-macroscopic model described above yields, formally, on taking the second moment of $\underset{\sim}{q} \mapsto \psi(\underset{\sim}{q}, \underset{\sim}{x}, t)$, the fully macroscopic, Oldroyd-B model of visco-elastic flow (cf. Section 2.2 below). On the other hand, when $\varepsilon>0$, it results in a macroscopic model which can be thought of as a dissipative Oldroyd-B model. In this sense, the Hookean dumbbell model has a macroscopic closure: it is the Oldroyd-B model when $\varepsilon=0$, and a dissipative version of Oldroyd-B (cf. (2.16) below) when $\varepsilon>0$. Lions and Masmoudi [31] have shown the existence of global-in-time weak solutions to the Oldroyd-B model in the simplified corotational case in which the symmetric part of the velocity gradient ${\underset{\sim}{\sim}}_{x} \underset{\sim}{u}$ featuring in the macroscopic counterpart of the drag term $\underset{\sim}{\nabla_{q}} \cdot\left(\left({\underset{\sim}{\nabla}}_{x} u\right) q \underset{\sim}{q} \psi\right)$ in assumed to vanish. At the level of the Fokker-Planck equation (1.5), corotationality corresponds to replacing the velocity gradient $\underset{\approx}{\nabla} \underset{\sim}{u}$ in the drag term by its skew-symmetric part. The argument of Lions and Masmoudi [31] is based on exploiting the propagation in time of the compactness of the solution and the DiPernaLions [16] theory of renormalized solutions to linear hyperbolic equations with nonsmooth transport coefficients. It is not known if an identical global existence result for the Oldroyd-B model also 
holds in the absence of the crucial assumption that the drag term is corotational. In a more recent paper, [32], using similar techniques, Lions and Masmoudi continued this line of investigation and considered a coupled Navier-Stokes-Fokker-Planck model, again with a corotational drag term, though this time with FENE-type nonlinear spring potentials. Again, they established the existence of a global weak solution; see also the related preprint by Masmoudi [36].

We note in passing that in contrast with the case of Hookean dumbbells, the FENE model does not have an exact closure at the macroscopic level, though Du, Yu, and Liu [17] and Yu, Du, and Liu [48] have recently considered the analysis of approximate closures of the FENE model. Previously, El-Kareh and Leal [19] had proposed a macroscopic model, with added dissipation in the equation which governs the evolution of the conformation tensor $\underset{\sim}{A}(\underset{\sim}{x}, t):=\int_{D} \underset{\sim}{q} q^{\top} \psi(\underset{\sim}{x}, \underset{\sim}{q}, t) \mathrm{d} q$ in order to account for Brownian motion across streamlines; the model can be thought of as an approximate macroscopic closure of a FENE-type microscopic-macroscopic model with centre-of-mass diffusion.

Barrett, Schwab, and Süli [2] established the existence of global-in-time weak solutions to the coupled microscopic-macroscopic model $(1.1 \mathrm{a}-\mathrm{d})$ and (1.5) with $\varepsilon=0$, an $\underset{\sim}{x}$-mollified velocitygradient in the Fokker-Planck equation and an $\underset{\sim}{x}$-mollified probability density function $\psi$ in the Kramers expression, - admitting a large class of potentials $U$ (including the Hookean dumbbell model as well as general FENE-type models); in addition to these mollifications, $\underset{\sim}{u}$ in the $\underset{\sim}{x}$-convective term $\left(\underset{\sim}{u} \cdot \nabla_{x}\right) \psi$ in the Fokker-Planck equation was also mollified. Unlike Lions and Masmoudi [31], the arguments in [2] did not require the assumption that the drag term was corotational in the FENE case. The Helmholtz-Stokes mollification $S_{\alpha} \underset{\sim}{u}$, with mollification lengthscale $\alpha$, of the velocity field $\underset{\sim}{u}$ that was considered in [2] was stimulated by the Leray- $\alpha$ model of the incompressible Navier-Stokes equations (the viscous Camassa-Holm equations), proposed by Foias, Holm, and Titi [22]. In [2] the motivation for introducing the mollification was of purely technical nature: the need to rigorously justify the passage to the limit in the proof of existence of a weak solution, based on a compactness argument. Surprisingly (cf. [3]), the derivation (from first principles) of the coupled Navier-StokesFokker-Planck model does, in fact, result in a mollification of $\psi$ in the Kramers formula for the extra-stress tensor as well as of the velocity gradient in the Fokker-Planck equation, just as in [2], albeit the mollifiers turn out to be directional Friedrichs mollifiers rather than Helmholtz-Stokes mollifiers. In classical derivations of the model the mollifiers are approximated by identity operators, on the grounds that the functions to which they are applied are smooth enough to justify such a model-reduction; absurdly, in the proof of existence of weak solutions to the resulting reduced model, the mollifiers then have to be reinstated since the requisite smoothness hypotheses which were used to justify the model-reduction are absent. In the present paper the mollifiers have been, nevertheless, replaced by identity operators. The rigorous mathematical justification of this model-reduction was given in our paper [3]: we showed there that in the case of the corotational model, with $\varepsilon>0$, weak solutions of the Friedrichs-mollified model converge to weak solutions of the associated model in which the Friedrichs mollifiers have been replaced by identity operators.

In a recent paper Jourdain, Lelièvre, and Le Bris [26] studied the existence of solutions to the FENE model in the case of a simple Couette flow; by using tools from the theory of stochastic differential equations, they established the existence of a unique local-in-time solution to the FENE model in two space dimensions $(d=2)$ when the velocity field $\underset{\sim}{u}$ is unidirectional and of the particular form $\underset{\sim}{u}\left(x_{1}, x_{2}\right)=\left(u_{1}\left(x_{2}\right), 0\right)^{\top}$. The notion of solution for which existence is proved in the paper of Jourdain, Lelièvre, and Le Bris [26] is mixed deterministic-stochastic in the sense that it is deterministic in the 'macroscopic' variable $\underset{\sim}{x}$, but stochastic in the 'microscopic' variable $q$. In contrast, our notion of solution (cf. Section 2 below) is deterministic both macroscopically and microscopically, since the microscales are modelled here by the probability density function $\psi(\underset{\sim}{x}, \underset{\sim}{q}, t)$. As has been noted in [2], the choice between these different notions of solution has far-reaching consequences on computational simulation: mixed deterministic-stochastic notions of solution necessitate the use of Monte Carlo-type algorithms for the numerical approximation of polymer configurations, as proposed in 
the monograph of Öttinger [39] and, for example, in the paper of Jourdain, Lelièvre, and Le Bris [25]; whereas weak solutions in the sense considered in the present paper can be approximated by entirely deterministic (e.g. Galerkin-type) schemes, as was done, for example, in Lozinski, Chauvière, Fang, and Owens [34]; see also [35]. Having said this, the probability density function $\psi$ depends on $2 d+1$ independent variables, the components of $\underset{\sim}{x}$ and $q$ and on $t$, so the implied computational cost is nontrivial; see, however, the recent papers by Süli [44] and Schwab, Süli, and Todor [45] concerning the sparse finite element approximation of high-dimensional transport-dominated diffusion equations. For a detailed survey of recent developments concerning stochastic and deterministic numerical techniques for kinetic models of polymers we refer to Chapter 11 in the book of Owens and Phillips [40], the monograph of Öttinger [39] and to Section 4 in the review article of $\mathrm{Li}$ and Zhang [30]. As has been noted by the authors of [30], numerical algorithms based on the kinetic theory of complex fluids have been vigorously developed since the 1970s; however, since both stochastic and deterministic numerical techniques for kinetic polymer models are computationally intensive, the numerical simulation of polymeric fluids via kinetic models represents a formidable computational task. Notwithstanding the computational cost, multiscale models of this type avoid potentially crude closure approximations and are therefore more accurate than classical macroscopic models. Hence, the development and analysis of numerical algorithms for kinetic models of dilute polymers represent mathematical challenges of considerable practical importance.

In this paper, we shall be concerned with the construction and convergence analysis of a general class of Galerkin methods for a corotational Navier-Stokes-Fokker-Planck dumbbell model with centre-of-mass diffusion. The paper is organized as follows. Section 2 is devoted to the statement of the problem. After listing our structural assumptions on the admissible class of nonlinear spring potentials, we define, in Section 3, a family of Galerkin-type methods for the coupled Navier-StokesFokker-Planck system, under very general assumptions on the finite-dimensional spaces used for the purpose of spatial discretization; our hypotheses admit a wide range of Galerkin subspaces, including, in particular, classical conforming finite element spaces (cf. Remark 3.5 for details). We show the existence and uniqueness of the associated numerical solution, and then pass to the limit as the spatial discretization parameter $h$ and the time step $\Delta t$ tend to zero; we use a weakcompactness argument to show that a subsequence of the sequence $\left\{\left(u_{\sim}^{\Delta t}, \widehat{\psi}_{h}^{\Delta t}\right)\right\}_{h>0, \Delta t>0}$ of numerical approximations to the velocity field $u$ and the scaled probability density function $\widehat{\psi}=\psi / M$, where $M$ is the normalized Maxwellian $M(\underset{\sim}{q})=C_{0} \exp \left(-U\left(\frac{1}{2}|q|\right)\right.$ ) with the (positive) constant $C_{0}$ chosen so that $\int_{D} M(q) \mathrm{d} q=1$, converges to a weak solution $(\underset{\sim}{\sim}, \widehat{\psi})$ of the coupled Navier-Stokes-FokkerPlanck system. This passage to the limit is performed under minimal assumptions on the data, and it therefore also provides a new proof of global existence of weak solutions to the corotational FENE model with centre-of-mass diffusion. Our mathematical machinery is less involved than the propagation-of-compactness argument used in the recent work of Lions and Masmoudi [32] mentioned above; in addition, the definition of the sequence of approximating solutions is completely constructive in the sense that it is based on a fully-discrete and practically implementable Galerkin method. To the best of our knowledge this is the first rigorous result concerning the convergence of a sequence of numerical approximations to a weak solution of the coupled Navier-Stokes-FokkerPlanck model. The convergence analysis of finite element approximations to the coupled NavierStokes-Fokker-Planck system with centre-of-mass diffusion in the general noncorotational case is the subject of our forthcoming paper [4].

2. Polymer Models. We term polymer models under consideration here microscopic-macroscopic type models, since the continuum mechanical macroscopic equations of incompressible fluid flow are coupled to a microscopic model: the Fokker-Planck equation describing the statistical properties of particles in the continuum. We first present these equations and collect the assumptions on the parameters in the model. 
2.1. Microscopic-Macroscopic Polymer Models. Let $\Omega \subset \mathbb{R}^{d}$ be a bounded open set with a Lipschitz-continuous boundary $\partial \Omega$, and suppose that the set $D \subseteq \mathbb{R}^{d}, d=2$ or 3 , of admissible elongation vectors $\underset{\sim}{q}$ in (1.5) is a balanced convex open set. For the sake of simplicity of presentation, we shall suppose that $D$ is a bounded open ball in $\mathbb{R}^{d}$ or the whole of $\mathbb{R}^{d}$. Gathering $(1.1 \mathrm{a}-\mathrm{d}),(1.3)$ and (1.5), we then consider the following initial-boundary-value problem:

(P) Find $\underset{\sim}{u}:(\underset{\sim}{x}, t) \in \mathbb{R}^{d+1} \mapsto \underset{\sim}{u}(\underset{\sim}{x}, t) \in \mathbb{R}^{d}$ and $p:(\underset{\sim}{x}, t) \in \mathbb{R}^{d+1} \mapsto p(\underset{\sim}{x}, t) \in \mathbb{R}$ such that

$$
\begin{aligned}
& \frac{\partial \tilde{\tau}}{\partial t}+\left(\underset{\sim}{u} \cdot \nabla_{x}\right) \underset{\sim}{u}-\nu \Delta_{x} \underset{\sim}{u}+\underset{\sim}{\nabla_{x}} p=\underset{\sim}{\nabla_{x}} \cdot \underset{\sim}{\tau}(\psi) \quad \text { in } \Omega \times(0, T], \\
& \underset{\sim}{\nabla_{x}} \cdot \underset{\sim}{u}=0 \quad \text { in } \Omega \times(0, T], \\
& \underset{\sim}{u}=\underset{\sim}{0} \quad \text { on } \partial \Omega \times(0, T], \\
& \underset{\sim}{u}(\underset{\sim}{x}, 0)={\underset{\sim}{u}}^{0}(\underset{\sim}{x}) \quad \forall \underset{\sim}{\forall} \in \Omega ;
\end{aligned}
$$

where $\nu \in \mathbb{R}_{>0}$ is the viscosity and $\underset{\sim}{\tau}(\psi):(\underset{\sim}{x}, t) \in \mathbb{R}^{d+1} \mapsto \underset{\sim}{\tau}(\psi)(\underset{\sim}{x}, t) \in \mathbb{R}^{d \times d}$ is the symmetric extrastress tensor, dependent on a probability density function $\psi:(\underset{\sim}{\sim}, \underset{\sim}{q}, t) \in \mathbb{R}^{2 d+1} \mapsto \psi(\underset{\sim}{x}, \underset{\sim}{q}, t) \in \mathbb{R}$, defined as

$$
\underset{\approx}{\tau}(\psi)=k \mu(\underset{\approx}{C}(\psi)-\rho(\psi) \underset{\approx}{I}) .
$$

Here $k, \mu \in \mathbb{R}_{>0}$ are, respectively, the Boltzmann constant and the absolute temperature, $\underset{\approx}{I}$ is the unit $d \times d$ tensor,

$$
\begin{array}{rlrl} 
& & \underset{\sim}{C}(\psi)\left({\underset{\sim}{x}}_{x}, t\right) & =\int_{D} \psi(\underset{\sim}{x}, \underset{\sim}{q}, t) U^{\prime}\left(\frac{1}{2}|\underset{\sim}{q}|^{2}\right) \underset{\sim}{q} \underset{\sim}{q^{\top}} \mathrm{d} \underset{\sim}{q} \\
\text { and } \quad & \rho(\psi)(\underset{\sim}{x}, t) & =\int_{D} \psi(\underset{\sim}{x}, \underset{\sim}{q}, t) \mathrm{d} \underset{\sim}{q} .
\end{array}
$$

In addition, the real-valued, continuous, nonnegative and strictly monotonic increasing function $U$, defined on a relatively open subset of $[0, \infty)$, is an elastic potential which gives the elastic force $\underset{\sim}{F}: D \rightarrow \mathbb{R}^{d}$ on the springs via (1.2).

The probability density $\psi(\underset{\sim}{x}, \underset{\sim}{q}, t)$ represents the probability at time $t$ of finding the centre of mass of a dumbbell in the volume element $\underset{\sim}{x}+\mathrm{d} x \underset{\sim}{x}$ and having the endpoint of its elongation vector within the volume element $q+\mathrm{d} q$. Hence $\rho(\psi)(x, t)$ is the density of the polymer chains located at $\underset{\sim}{x}$ at time $t$. The function $\widetilde{\psi}$ satisfies the following Fokker-Planck equation, together with suitable boundary and initial conditions:

$$
\begin{aligned}
& \left.\left.\frac{\partial \psi}{\partial t}+\underset{\sim}{u} \cdot \underset{\sim}{\nabla} x\right) \psi+\underset{\sim}{\nabla_{q}} \cdot \underset{\sim}{(\sigma} \underset{\sim}{u} \underset{\sim}{q} \psi\right)=\frac{1}{2 \lambda} \underset{\sim}{\nabla_{q}} \cdot\left(\underset{\sim}{\nabla_{q}} \psi+U^{\prime} \underset{\sim}{q} \psi\right)+\varepsilon \Delta_{x} \psi \quad \text { in } \Omega \times D \times(0, T], \\
& \psi=0 \quad \text { on } \Omega \times \partial D \times(0, T], \\
& \varepsilon \underset{\sim}{\nabla_{x} \psi \cdot n} \underset{\sim}{n}=0 \quad \text { on } \partial \Omega \times D \times(0, T], \\
& \psi(\underset{\sim}{x}, \underset{\sim}{q}, 0)=\psi^{0}(\underset{\sim}{x}, \underset{\sim}{q}) \geq 0 \quad \forall(\underset{\sim}{x}, \underset{\sim}{q}) \in \Omega \times D
\end{aligned}
$$

where $\underset{\sim}{n}$ is the unit outward normal vector to $\partial \Omega$. When $D=\mathbb{R}^{d}$, the boundary condition (2.4b) on $\partial D$, the boundary of $D$, is replaced by a decay condition at infinity which demands that $|\psi|$ converges to 0 sufficiently fast as $|\underset{\sim}{q}|$ tends to $\infty$ (cf. [3]).

In (2.4a) the parameter $\lambda \in \widetilde{\mathbb{R}}_{>0}$ characterizes the elastic relaxation property of the fluid, and $\underset{\sim}{\sigma(v)})$ is related to $\underset{\sim}{\underset{\sim}{\nabla}} \underset{\sim}{v}$, where $(\underset{\sim}{\underset{\sim}{\nabla}} \underset{\sim}{v})(\underset{\sim}{x}, t) \in \mathbb{R}^{d \times d}$ and $\{\underset{\approx}{\underset{\sim}{\nabla}} \underset{\sim}{v}\}_{i j}=\frac{\partial v_{i}}{\partial x_{j}}$. The analytical results in this paper are concerned with the corotational model, where

$$
\underset{\approx}{\sigma}(\underset{\sim}{v}):=\underset{\sim}{\omega}(\underset{\sim}{v})
$$


here

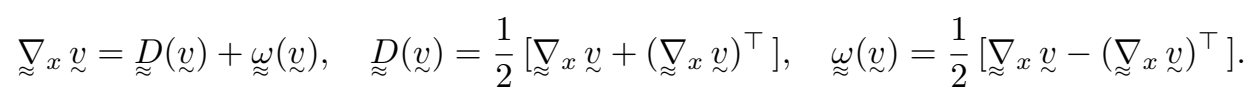

On introducing the (normalized) Maxwellian

$$
M(\underset{\sim}{q})=\frac{\mathrm{e}^{-U\left(\frac{1}{2}|q|^{2}\right)}}{\int_{D} \mathrm{e}^{-U} \mathrm{~d} q}
$$

we have that

$$
M \underset{\sim}{\underset{q}{\nabla}} M^{-1}=-M^{-1} \underset{\sim}{\underset{q}{\nabla}} M=U^{\prime} \underset{\sim}{q}
$$

In addition, the following identities hold:

$$
\nabla_{q} U=U^{\prime} \underset{\sim}{q}, \quad \nabla_{q} U^{\prime}=U^{\prime \prime} \underset{\sim}{q} \quad \text { and } \quad \Delta_{q} U=U^{\prime \prime}|\underset{\sim}{q}|^{2}+U^{\prime} d .
$$

Thus, the Fokker-Planck equation (2.4a) can be rewritten as

$$
\frac{\partial \psi}{\partial t}+\left(\underset{\sim}{u} \cdot \underset{\sim}{\nabla_{x}}\right) \psi+\underset{\sim}{\nabla_{q}} \cdot(\underset{\sim}{\sigma(u)} \underset{\sim}{q} \psi)=\frac{1}{2 \lambda} \nabla_{q} \cdot\left(M \underset{\sim}{\nabla_{q}}\left(\frac{\psi}{M}\right)\right)+\varepsilon \Delta_{x} \psi \quad \text { in } \Omega \times D \times(0, T] .
$$

2.2. Two Examples. We present two relevant examples of spring potentials: the FENE potential, where $D$ is a bounded open ball in $\mathbb{R}^{d}$, and the case of a Hookean potential, where $D=\mathbb{R}^{d}$.

2.2.1. FENE-type models. A widely used model is the FENE model, where

$$
D=B\left(\underset{\sim}{0}, b^{\frac{1}{2}}\right) \quad \text { and } \quad U(s)=-\frac{b}{2} \ln \left(1-\frac{2 s}{b}\right), \quad \text { and hence } \quad \mathrm{e}^{-U\left(\frac{1}{2}|q|^{2}\right)}=\left(1-\frac{|q|^{2}}{b}\right)^{\frac{b}{2}} .
$$

Here $B(\underset{\sim}{0}, s)$ is the bounded open ball of radius $s>0$ in $\mathbb{R}^{d}$ centred at the origin, and $b>0$ is an input parameter. Hence the length $|\underset{\sim}{\mid}|$ of the elongation vector $\underset{\sim}{q}$ cannot exceed $b^{\frac{1}{2}}$.

2.2.2. Hookean dumbbells. Letting $b \rightarrow \infty$ in (2.10) leads to the so-called Hookean dumbbell model where

$$
D=\mathbb{R}^{d} \quad \text { and } \quad U(s)=s, \quad \text { and therefore } \quad \mathrm{e}^{-U\left(\frac{1}{2}|q|^{2}\right)}=\mathrm{e}^{-\frac{1}{2}|q|^{2}} .
$$

This particular kinetic model, with $\varepsilon \in \mathbb{R}_{>0}$ and $\underset{\widetilde{u}}{\sigma}(\underset{\sim}{u})=\nabla_{x} \underset{\sim}{u}$, corresponds formally to a dissipative Oldroyd-B type model, or with $\varepsilon \in \mathbb{R}_{>0}$ and $\left.\underset{\sim}{\sigma(\widetilde{\sim}}\right)=\underset{\sim}{(\underset{\sim}{\sim}(\tilde{u})}$ to a corotational dissipative Oldroyd-B type model. Indeed, on multiplying (2.4a) by $\underset{\sim}{q} \underset{\sim}{q}$, integrating over $D$, performing integration by parts (assuming that $\psi$ and $\left|\nabla_{q} \psi\right|$ decay to zero sufficiently fast as $|\underset{\sim}{\sim}| \rightarrow \infty$ ), and noting (2.3a) and for any $\underset{\sim}{r} \in \mathbb{R}^{d}$ that

$$
\left(\underset{\sim}{r} \cdot \nabla_{q}\right) \underset{\sim}{q}{\underset{\sim}{q}}^{\top}=\underset{\sim}{r} \sim^{\top}+\underset{\sim}{q}{\underset{\sim}{r}}^{\top} \quad \text { and } \quad \Delta_{q}\left(\underset{\sim}{q} \sim^{\top}\right)=2 \underset{\sim}{I},
$$

yields that $\underset{\approx}{C} \underset{\sim}{x}, t) \equiv \underset{\sim}{C}(\psi(\underset{\sim}{x}, t))$ satisfies

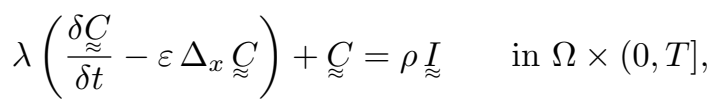


where $\rho(\underset{\sim}{x}, t) \equiv \rho(\psi(\underset{\sim}{x}, t))$ and

$$
\frac{\delta \underset{\approx}{\delta t}}{\delta t} \frac{\partial \underset{\approx}{\partial t}}{\partial t}+(\underset{\sim}{u} \cdot \underset{\sim}{\nabla}) \underset{\approx}{C}-\left[\underset{\approx}{\sigma}(u) \underset{\approx}{C}+\underset{\approx}{C}[\underset{\approx}{\sigma}(u)]^{\top}\right]
$$

is the upper-convected time derivative. Similarly, on integrating (2.4a) over $D$ and noting (2.3b) yields that $\rho$ satisfies

$$
\frac{\partial \rho}{\partial t}-\varepsilon \Delta_{x} \rho+\left(\underset{\sim}{u} \cdot \nabla_{x}\right) \rho=0 \quad \text { in } \Omega \times(0, T] .
$$

Hence in the Hookean case, the probability density function $\psi$ can be eliminated, leading to a closed model for $\underset{\sim}{u}, \underset{\approx}{C}$ and $\rho$. Moreover, in the case of the corotational model, (2.13) and (2.15) can be combined to yield that the extra-stress tensor $\underset{\sim}{\tau}(\psi)$ satisfies

$$
\lambda\left(\frac{\delta \underset{\approx}{\tau t}}{\delta t}-\varepsilon \Delta_{x} \underset{\approx}{\tau}\right)+\underset{\approx}{\tau}=k \mu \lambda \rho\left[\underset{\sim}{\sigma}(u)+[\underset{\sim}{\sigma}(u)]^{\top}\right] \quad \text { in } \Omega \times(0, T]
$$

which, in the case of formally setting $\varepsilon=0$, is the Oldroyd-B constitutive equation if $\underset{\sim}{\sigma}(\underset{\sim}{u})=\underset{\approx}{\underset{\sim}{\nabla}} \underset{\sim}{u}$ or the corotational Oldroyd-B constitutive equation if $\underset{\sim}{\sigma(u)}(\underset{\sim}{2}) \underset{\sim}{\omega}(u)$; in the latter case, the right-hand side of (2.16) is identically equal to 0 . With $\varepsilon>0,(2.16)$ represents dissipative versions of these Oldroyd-B models.

2.3. General Structural Assumptions on the Potential. As has been noted in the Introduction, the choice of $D=\mathbb{R}^{d}$ (corresponding to the Hookean model) is physically unrealistic; thus, we shall henceforth suppose for simplicity that $D$ is a bounded open ball in $\mathbb{R}^{d}$. We assume that

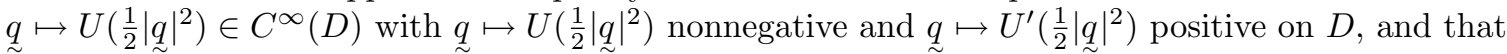
there exis $\widetilde{t}$ constants $c_{i}>0, \widetilde{i}=1,2,3,4$, such that the Maxwellian $\widetilde{M}$ and the associated elastic potential $U$ satisfy

$$
\begin{gathered}
c_{1}[\operatorname{dist}(\underset{\sim}{q}, \partial D)]^{\kappa} \leq M(\underset{\sim}{q}) \leq c_{2}[\operatorname{dist}(\underset{\sim}{q}, \partial D)]^{\kappa} \quad \forall \underset{\sim}{q} \in D, \\
c_{3} \leq[\operatorname{dist}(\underset{\sim}{q}, \partial D)] U^{\prime}\left(\frac{1}{2}|\underset{\sim}{q}|^{2}\right) \leq c_{4} \quad \forall \underset{\sim}{q} \in D .
\end{gathered}
$$

It is an easy matter to show that the Maxwellian $M$ and the elastic potential $U$ of the FENE dumbbell model satisfy conditions $(2.17 \mathrm{a}, \mathrm{b})$ with $D=B\left(\underset{\sim}{0}, b^{\frac{1}{2}}\right)$ and $\kappa=\frac{b}{2}$.

Since $[U(\underset{\sim}{q})]^{2}=(-\ln M(\underset{\sim}{q})+\text { Const. })^{2}$, it follows from $(2.17 \mathrm{a})$ and $(2.17 \mathrm{~b})$ that if $\kappa>1$, then

$$
\int_{D}\left[1+U^{2}+\left|U^{\prime}\right|^{2}\right] M \underset{\sim}{\mathrm{d} q}<\infty .
$$

We shall therefore suppose that $\kappa>1$. For the FENE model $(2.10), \kappa=\frac{b}{2}$, and so the condition $\kappa>1$ translates into the requirement that $b>2$. It is interesting to note that in the, equivalent, stochastic version of the FENE model, a solution to the system of stochastic differential equations associated with the Fokker-Planck equation exists and has trajectorial uniqueness if, and only if, $b>2$ (cf. [26] for details). Thus, the assumption $\kappa>1$ can be seen as the weakest reasonable requirement on the decay-rate of $M$ as $\operatorname{dist}(\underset{\sim}{q}, \partial D) \rightarrow 0$.

3. Numerical Approximation. Assuming that $\partial \Omega \in C^{0,1}$, let

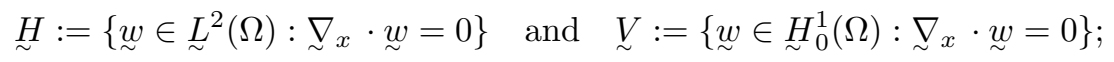

where the divergence operator $\nabla_{x}$. is to be understood in the sense of vector-valued distributions

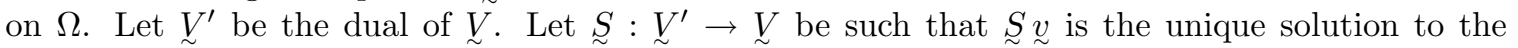
Helmholtz-Stokes problem

$$
\int_{\Omega} \underset{\sim}{S} \underset{\sim}{v} \cdot \underset{\sim}{w} \mathrm{~d} x+\int_{\Omega} \underset{\sim}{\nabla} \underset{\sim}{S} \underset{\sim}{v}: \underset{\sim}{\nabla_{x}} \underset{\sim}{w} \mathrm{~d} x=\langle\underset{\sim}{v}, \underset{\sim}{w}\rangle \quad \forall \underset{\sim}{w} \in \underset{\sim}{V}
$$


where $\langle\cdot, \cdot\rangle$ denotes the duality pairing between $\underset{\sim}{V^{\prime}}$ and $\underset{\sim}{V}$. We note that

$$
\langle\underset{\sim}{v}, \underset{\sim}{\underset{\sim}{v}\rangle}\rangle=\|\underset{\sim}{S} \underset{\sim}{v}\|_{H^{1}(\Omega)}^{2} \quad \forall \underset{\sim}{v} \in V^{\prime} \supset\left({\underset{\sim}{H}}_{0}^{1}(\Omega)\right)^{\prime},
$$

and $\|S \cdot\|_{H^{1}(\Omega)}$ is a norm on $V^{\prime}$.

On introducing

$$
\|\widehat{\varphi}\|_{H^{1}(\Omega \times D ; M)}:=\left\{\int_{\Omega \times D} M\left[|\widehat{\varphi}|^{2}+\left|\nabla_{x} \widehat{\varphi}\right|^{2}+\left|\nabla_{q} \widehat{\varphi}\right|^{2}\right] \mathrm{d} q \underset{\sim}{\mathrm{d} x}\right\}^{\frac{1}{2}}
$$

we then set

$$
\widehat{X} \equiv H^{1}(\Omega \times D ; M):=\left\{\widehat{\varphi} \in L_{\mathrm{loc}}^{1}(\Omega \times D):\|\widehat{\varphi}\|_{H^{1}(\Omega \times D ; M)}<\infty\right\} .
$$

It follows that

$$
C^{\infty}(\overline{\Omega \times D}) \text { is dense in } \widehat{X} \text {. }
$$

This can be shown, for example, by a simple adaption of Lemma 3.1 in Barrett, Schwab, and Süli [2], which appeals to fundamental results on weighted Sobolev spaces in Triebel [47] and Kufner [28].

We note for future reference that (2.3a) and (2.18) yield for $M^{\frac{1}{2}} \widehat{\varphi} \in L^{2}(\Omega \times D)$ that

$$
\begin{aligned}
& \int_{\Omega}|C(M \widehat{\sim})|^{2} \mathrm{~d} x=\int_{\Omega} \sum_{i=1}^{d} \sum_{j=1}^{d}\left(\int_{D} M \widehat{\varphi} U^{\prime} q_{i} q_{j} \underset{\sim}{\mathrm{d} q}\right)^{2} \mathrm{~d} \underset{\sim}{\sim} \\
& \leq d\left(\left.\int_{D} M\left(U^{\prime}\right)^{2} \underset{\sim}{\mid q}\right|^{4} \mathrm{~d} q\right)\left(\int_{\Omega \times D} M|\widehat{\varphi}|^{2} \underset{\sim}{\mathrm{d} q} \mathrm{~d} x\right) \\
& \leq C\left(\int_{\Omega \times D} M|\widehat{\varphi}|^{2} \underset{\sim}{\mathrm{d}} \underset{\sim}{\mathrm{d} x}\right),
\end{aligned}
$$

where $C=C(d)$ is a positive constant.

We note that

$$
\underset{\sim}{\omega}(\underset{\sim}{v})=-[\underset{\sim}{\omega}(v)]^{\top} \text { and hence } \underset{\sim}{q^{\top}} \underset{\sim}{\omega}(\underset{\sim}{v}) \underset{\sim}{q}=0 \quad \forall \underset{\sim}{q} \in \mathbb{R}^{d} .
$$

On recalling $(4.15 \mathrm{a}, \mathrm{b})$ in Barrett, Schwab, and Süli [2], it follows for all $\underset{\sim}{v} \in W^{1, \infty}(\Omega)$ and $\widehat{\varphi} \in \widehat{X}$ that

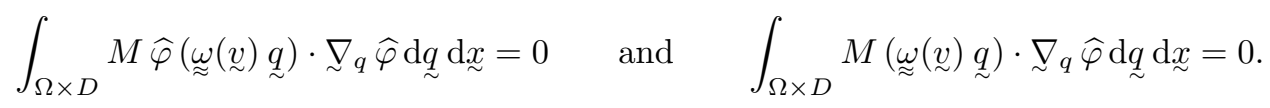

The aim of this paper is construct a numerical approximation of $(\mathbf{P})$; and prove convergence of this approximation, and hence prove existence of a (global-in-time) solution to the following weak formulation $\left(\mathbf{P}_{\mathbf{w}}\right)$ of $(\mathbf{P})$, with $\widehat{\psi}=\psi / M$, for given initial data

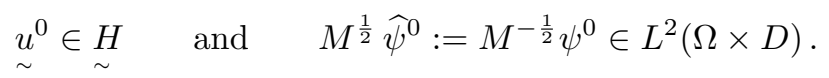

$\left(\mathbf{P}_{\mathbf{w}}\right)$ Find $\underset{\sim}{u} \in L^{\infty}\left(0, T ; L^{2}(\Omega)\right) \cap L^{2}(0, T ; \underset{\sim}{V}) \cap W^{1, \frac{4}{\beta}}\left(0, T ; V_{\sim}^{\prime}\right)$ and $\widehat{\psi} \in L^{2}(0, T ; \widehat{X})$, with $M^{\frac{1}{2}} \widehat{\psi} \in$ 
$L^{\infty}\left(0, T ; L^{2}(\Omega \times D)\right)$ and $\underset{\approx}{C}(M \widehat{\psi}) \in L^{\infty}\left(0, T ; \underset{\sim}{L^{2}}(\Omega)\right)$, such that $\underset{\sim}{u}(\cdot, 0)=u_{\sim}^{0}(\cdot)$ and

$$
\begin{aligned}
& \int_{0}^{T}\left\langle\frac{\partial \widetilde{\sim}}{\partial t}, \underset{\sim}{w}\right\rangle \mathrm{d} t+\int_{0}^{T} \int_{\Omega}\left[\left[\left(\underset{\sim}{u} \cdot \nabla_{x}\right) u\right] \cdot \underset{\sim}{w}+\nu \nabla_{x} \underset{\sim}{u}:_{\sim}^{\nabla_{x}} \underset{\sim}{w}\right] \mathrm{d} x \mathrm{~d} t \\
& =-\int_{0}^{T} \int_{\Omega} \underset{\approx}{C}(M \widehat{\psi}): \underset{\approx}{\underset{\sim}{w}} \underset{\sim}{\mathrm{d} x} \mathrm{~d} t \quad \forall \underset{\sim}{w} \in L^{\frac{4}{4-\beta}}(0, T ; \underset{\sim}{V}) ; \\
& -\int_{0}^{T} \int_{\Omega \times D} M \widehat{\psi} \frac{\partial \widehat{\varphi}}{\partial t} \mathrm{~d} q \underset{\sim}{\mathrm{d} x} \mathrm{~d} t-\int_{\Omega \times D} M \widehat{\psi}^{0}(\cdot, \cdot) \widehat{\varphi}(\cdot, \cdot, 0) \mathrm{d} \underset{\sim}{q} \mathrm{~d} x \\
& +\int_{0}^{T} \int_{\Omega \times D}\left\{\left[\varepsilon M \nabla_{x} \widehat{\psi}-\underset{\sim}{u} \widehat{\psi}\right] \cdot \nabla_{x} \widehat{\varphi}+\frac{M}{2 \lambda} \underset{\sim}{\nabla_{q}} \widehat{\psi} \cdot \underset{\sim}{\nabla_{q} \widehat{\varphi}}\right\} \mathrm{d} q \underset{\sim}{\mathrm{d} x} \mathrm{~d} t \\
& +\frac{1}{2} \int_{0}^{T} \int_{\Omega \times D}\left[\left[\nabla_{x}\left(M \widehat{\psi} \nabla_{q} \widehat{\varphi}\right) \underset{\sim}{q}\right] \cdot \underset{\sim}{u}-(\underset{\sim}{u} \cdot \underset{\sim}{q})\left[\nabla_{x} \cdot\left(M \widehat{\psi} \nabla_{q} \widehat{\varphi}\right)\right]\right] \mathrm{d} \underset{\sim}{q} \mathrm{~d} x \underset{\sim}{\mathrm{d} t} t=0 \\
& \forall \widehat{\varphi} \in \widehat{\mathcal{X}}
\end{aligned}
$$

where

$$
\beta \in(2,4) \quad \text { if } d=2 \quad \text { and } \quad \beta=3 \quad \text { if } d=3,
$$

and $\widehat{\mathcal{X}}$ is defined as the completion of $C_{0}^{\infty}\left((-T, T) ; C^{\infty}(\overline{\Omega \times D})\right)$ in the norm $\|\cdot\|_{\widehat{\mathcal{X}}}$ defined by

$$
\|\widehat{\varphi}\|_{\widehat{\mathcal{X}}}:=\|\widehat{\varphi}\|_{L^{2}(0, T ; \widehat{X})}+\left\|M^{\frac{1}{2}} \underset{\sim}{\nabla_{q}} \widehat{\varphi}\right\|_{L^{2}\left(0 ; T ; H^{1}\left(\Omega ; L^{2}(D)\right)\right)}+\left\|M^{\frac{1}{2}} \frac{\partial \widehat{\varphi}}{\partial t}\right\|_{L^{2}\left(0 ; T ; L^{2}(\Omega \times D)\right)} .
$$

This, in particular, implies that each $\widehat{\varphi} \in \widehat{\mathcal{X}}$ satisfies $\widehat{\varphi}(\cdot, \cdot, T)=0$.

REMARK 3.1. The imposition of the initial condition to the u-equation is understood in the sense that $\lim _{t \rightarrow 0} \int_{\Omega}\left[\underset{\sim}{u}(\cdot, t)-{\underset{\sim}{u}}^{0}(\cdot)\right] \cdot \underset{\sim}{v}(\cdot) \mathrm{d} \underset{\sim}{x}=0$ for all $\underset{\sim}{v} \in \underset{\sim}{H}$, see e.g. Theorem 3.1 on $p .191$ in Temam [46].

REMARK 3.2. Since the test functions in $V$ are divergence-free, the pressure has been eliminated in (3.11a,b); it can be recovered in a very weak sense following the same procedure as for the incompressible Navier-Stokes equations discussed on p.208 in Temam [46]; i.e., one obtains that $\int_{0}^{t} p(\cdot, s) \mathrm{d} s \in C\left([0, T] ; L^{2}(\Omega)\right)$.

Let $h>0$ denote a discretization parameter tending to zero and, for each $h$, let $W_{h}$ and $R_{h}$ be two finite-dimensional spaces such that

$$
\underset{\sim}{W} \subset \underset{\sim}{H} H_{0}^{1}(\Omega) \cap \underset{\sim}{W^{1, \infty}}(\Omega) \quad \text { and } \quad R_{h} \subset L_{0}^{2}(\Omega):=\left\{r \in L^{2}(\Omega): \int_{\Omega} r \underset{\sim}{r} \underset{\sim}{\operatorname{d}} \mathrm{d} x=0\right\} .
$$

We assume that $\bigcup_{h>0} \underset{\sim}{W}$ and $\bigcup_{h>0} R_{h}$ are dense in $\underset{\sim}{H}(\Omega)$ and $L_{0}^{2}(\Omega)$, respectively. In addition, we set

$$
\underset{\sim}{V_{h}}:=\left\{\underset{\sim}{w_{h}} \in \underset{\sim}{W_{h}}: \int_{\Omega}\left(\underset{\sim}{\nabla_{x}} \cdot \underset{\sim}{w_{h}}\right) r_{h} \mathrm{~d} x=0 \quad \forall r_{h} \in R_{h}\right\} .
$$

Moreover, we assume the inf-sup condition; that is, there exists a positive constant $C_{0}$, independent of $h$, such that

$$
\sup _{\sim} \underset{\sim}{w_{h} \in W_{h}} \frac{\int_{\Omega}\left(\underset{\sim}{\nabla} \sim_{x} \cdot{\underset{\sim}{w}}_{h}\right) r_{h} \mathrm{~d} x}{\left\|\sim_{\sim}\right\|_{H^{1}(\Omega)}} \geq C_{0}\left\|r_{h}\right\|_{L^{2}(\Omega)} \quad \forall r_{h} \in R_{h} .
$$


Hence it follows that

$$
\forall \underset{\sim}{\forall} \in \underset{\sim}{V}, \quad \exists\left\{\underset{\sim}{v_{h}}\right\}_{h>0}, \underset{\sim}{v_{h}} \in \underset{\sim}{V_{h}}, \quad \text { such that } \quad \lim _{h \rightarrow 0}\left\|\underset{\sim}{v}-\underset{\sim}{v_{h}}\right\|_{H^{1}(\Omega)}=0
$$

We require the $L^{2}$ projector $Q_{h}: \underset{\sim}{V} \rightarrow \underset{\sim}{V}$ defined by

$$
\int_{\Omega}\left(\underset{\sim}{v}-\underset{\sim}{Q_{h}} \underset{\sim}{v}\right) \cdot \underset{\sim}{w_{h}} \mathrm{~d} \underset{\sim}{x}=0 \quad \forall \underset{\sim}{w_{h}} \in \underset{\sim}{V_{h}}
$$

We shall assume that $\Omega$ and $\underset{\sim}{V}$ are such that this projection is uniformly $H^{1}$ stable; that is,

$$
\left\|\underset{\sim}{Q_{h}} \underset{\sim}{v}\right\|_{H^{1}(\Omega)} \leq C_{1}\|\underset{\sim}{v}\|_{H^{1}(\Omega)} \quad \forall \underset{\sim}{v} \in \underset{\sim}{V} .
$$

REMARK 3.3. In the case of Galerkin finite element approximations on quasi-uniform families of partitions into simplices of $\Omega \subset \mathbb{R}^{d}, d=2,3$, where $\Omega$ is either a $C^{2}$ domain or a convex polytope, the inequality (3.19) is known to hold (see Heywood and Rannacher [24]). On the other hand, for the $L^{2}$ projector onto $W_{h}(\supset \underset{\sim}{V})$, these hypotheses for ensuring uniform stability in the $H^{1}$ norm can be considerably relaxed; see, for example, [8, 11, 15]. As it is likely that the strong assumptions from [24] are not necessary for the $H^{1}$ stability of $Q_{h}$, we chose to adopt hypothesis (3.19) directly instead of imposing a demanding sufficient condition to ensure that it holds.

Let $\widehat{X}_{h}^{x} \subset W^{1, \infty}(\Omega)$ and $\widehat{X}_{h}^{q} \subset W^{1, \infty}(D)$ be finite-dimensional spaces of functions defined on $\bar{\Omega}$ and $\bar{D}$, respectively, such that the following standard approximation properties hold:

$$
\lim _{h \rightarrow 0} \operatorname{dist}_{W^{1, \infty}(\Omega)}\left(\eta, \widehat{X}_{h}^{x}\right)=0 \quad \forall \eta \in C^{\infty}(\bar{\Omega}), \quad \lim _{h \rightarrow 0} \operatorname{dist}_{W^{1, \infty}(D)}\left(\zeta, \widehat{X}_{h}^{q}\right)=0 \quad \forall \zeta \in C^{\infty}(\bar{D}) .
$$

In addition, we define

$$
W^{(1,1), \infty}(\Omega \times D):=\left\{\widehat{\varphi} \in L^{\infty}(\Omega \times D): \frac{\partial^{k}}{\partial x_{i}^{k}} \frac{\partial^{\ell}}{\partial q_{j}^{\ell}} \widehat{\varphi} \in L^{\infty}(\Omega \times D), \quad i, j=1 \rightarrow d, \quad k, \ell \in\{0,1\}\right\} .
$$

Lemma 3.4. Suppose that $\widehat{X}_{h}:=\widehat{X}_{h}^{x} \otimes \widehat{X}_{h}^{q} \subset \widehat{X} \cap W^{(1,1), \infty}(\Omega \times D)$, where $\widehat{X}_{h}^{x} \subset W^{1, \infty}(\Omega)$ and $\widehat{X}_{h}^{q} \subset W^{1, \infty}(D)$ are finite-dimensional spaces of functions defined on $\bar{\Omega}$ and $\bar{D}$, respectively, such that (3.20) holds. Then, for each $\widehat{\varphi} \in C^{\infty}(\overline{\Omega \times D})$ there exists a monotonic decreasing sequence of positive real numbers $\left\{h_{m}\right\}_{m \geq 1}$ converging to zero and elements $\left\{I_{h_{m}} \widehat{\varphi}\right\}_{m \geq 1}$, with $I_{h_{m}} \widehat{\varphi} \in \widehat{X}_{h_{m}}$, such that

$$
\lim _{m \rightarrow \infty}\left\|\frac{\partial^{k}}{\partial x_{i}^{k}} \frac{\partial^{\ell}}{\partial q_{j}^{\ell}}\left(\widehat{\varphi}-I_{h_{m}} \widehat{\varphi}\right)\right\|_{L^{\infty}(\Omega \times D)}=0, \quad i, j=1 \rightarrow d, \quad k, \ell \in\{0,1\} .
$$

Proof. Let $K_{0}$ and $K_{1}$ be compact subsets of $\mathbb{R}^{2 d}$ such that $\overline{\Omega \times D} \subset \subset K_{1} \subset \subset K_{0}$. Given $\widehat{\varphi} \in C^{\infty}(\overline{\Omega \times D})$, let $\widehat{\varphi}^{*} \in C^{2,1}\left(\mathbb{R}^{2 d}\right)$ denote the Whitney extension (cf. Stein [43]) of $\widehat{\varphi}$, considered as an element of $C^{2,1}(\overline{\Omega \times D})$, from $\overline{\Omega \times D}$ to $\mathbb{R}^{2 d}$, and let $\chi \in C_{0}^{\infty}\left(K_{0}\right)$ be such that $\chi \equiv 1$ on $K_{1}$. Then $\chi \widehat{\varphi}^{*} \in C_{0}^{2,1}\left(K_{0}\right) \subset C_{0}^{2}\left(K_{0}\right)$ and $\left.\left(\chi \widehat{\varphi}^{*}\right)\right|_{\overline{\Omega \times D}}=\widehat{\varphi}$. By Nachbin's variant of the StoneWeierstrass theorem (see [37] and Corollary 3 in [21]), for any $\delta>0$ there exists a nonnegative integer $n=n(\delta)$ and a polynomial $p_{n}(\underset{\sim}{x} \underset{\sim}{q})$ of degree $n$ defined on $\mathbb{R}^{2 d}$ such that

$$
\left\|\chi \widehat{\varphi}^{*}-p_{n}\right\|_{C^{2}\left(K_{0}\right)}<\delta .
$$


Hence,

$$
\max _{i, j \in\{1, \ldots, d\}} \max _{k, \ell \in\{0,1\}}\left\|\frac{\partial^{k}}{\partial x_{i}^{k}} \frac{\partial^{\ell}}{\partial q_{j}^{\ell}}\left(\widehat{\varphi}-p_{n}\right)\right\|_{L^{\infty}(\Omega \times D)} \leq\left\|\widehat{\varphi}-p_{n}\right\|_{C^{2}(\overline{\Omega \times D})}<\delta .
$$

Let us expand

$$
p_{n}(\underset{\sim}{x}, \underset{\sim}{q})=\sum_{\alpha, \gamma \in \mathbb{N}_{\geq 0}^{d}:|\alpha|+|\gamma| \leq n} a_{\alpha, \gamma}{\underset{\sim}{x}}_{x^{\alpha}}^{q^{\gamma}}
$$

where $\mathbb{N}_{\geq 0}^{d}$ denotes the $d$-fold cartesian product of the set $\mathbb{N}_{\geq 0}$ of nonnegative integers.

Then, as a consequence of (3.20), there exist functions $\eta_{\alpha, h} \in \widehat{X}_{h}^{x}$ and $\zeta_{\gamma, h} \in \widehat{X}_{h}^{q}$ such that

$$
\lim _{h \rightarrow 0}\left\|x^{\alpha}-\eta_{\alpha, h}\right\|_{W^{1, \infty}(\Omega)}=0 \quad \text { and } \quad \lim _{h \rightarrow 0}\left\|q^{\gamma}-\zeta_{\gamma, h}\right\|_{W^{1, \infty}(D)}=0, \quad \alpha, \gamma \in \mathbb{N}_{\geq 0}^{d}:|\alpha|+|\gamma| \leq n
$$

With $I_{h}^{\delta} \widehat{\varphi} \in \widehat{X}_{h}=\widehat{X}_{h}^{x} \otimes \widehat{X}_{h}^{q}$ defined, for $n=n(\delta)$, by

$$
I_{h}^{\delta} \widehat{\varphi}(\underset{\sim}{x}, \underset{\sim}{q}):=\sum_{\alpha, \gamma \in \mathbb{N}_{\geq 0}^{d}:|\alpha|+|\gamma| \leq n} a_{\alpha, \gamma} \eta_{\alpha, h} \zeta_{\gamma, h}
$$

we then deduce, for $\delta>0$ (and $n=n(\delta))$ fixed, that

$$
\lim _{h \rightarrow 0} \max _{i, j \in\{1, \ldots, d\}} \max _{k, \ell \in\{0,1\}}\left\|\frac{\partial^{k}}{\partial x_{i}^{k}} \frac{\partial^{\ell}}{\partial q_{j}^{\ell}}\left(p_{n}-I_{h}^{\delta} \widehat{\varphi}\right)\right\|_{L^{\infty}(\Omega \times D)}=0 .
$$

Combining the above and (3.22), with $\delta>0$ (and $n=n(\delta))$ fixed, then gives

$$
\lim _{h \rightarrow 0} \max _{i, j \in\{1, \ldots, d\}} \max _{k, \ell \in\{0,1\}}\left\|\frac{\partial^{k}}{\partial x_{i}^{k}} \frac{\partial^{\ell}}{\partial q_{j}^{\ell}}\left(\widehat{\varphi}-I_{h}^{\delta} \widehat{\varphi}\right)\right\|_{L^{\infty}(\Omega \times D)}<\delta,
$$

which, in turn, implies the existence of $h_{0}=h_{0}(\delta)>0$ such that

$$
\max _{i, j \in\{1, \ldots, d\}} \max _{k, \ell \in\{0,1\}}\left\|\frac{\partial^{k}}{\partial x_{i}^{k}} \frac{\partial^{\ell}}{\partial q_{j}^{\ell}}\left(\widehat{\varphi}-I_{h}^{\delta} \widehat{\varphi}\right)\right\|_{L^{\infty}(\Omega \times D)}<\delta \quad \forall h \in\left(0, h_{0}\right] .
$$

Thus we have shown that for each $\widehat{\varphi} \in C^{\infty}(\overline{\Omega \times D})$ and each $\delta>0$ there exists $I_{h}^{\delta} \widehat{\varphi} \in \widehat{X}_{h}$ and $h_{0}=$ $h_{0}(\delta)$ such that $(3.23)$ holds. Consider the sequence $\delta_{m}=1 / m, m=1,2, \ldots$, and let $\left\{h_{m}\right\}_{m \geq 1}$ be a monotonic decreasing sequence of positive real numbers converging to zero, with $h_{m} \in\left(0, h_{0}\left(\delta_{m}\right)\right]$. Letting

$$
I_{h_{m}} \widehat{\varphi}:=I_{h_{m}}^{\delta_{m}} \widehat{\varphi} \in \widehat{X}_{h_{m}}, \quad m=1,2, \ldots
$$

it then follows that

$$
\max _{i, j \in\{1, \ldots, d\}} \max _{k, \ell \in\{0,1\}}\left\|\frac{\partial^{k}}{\partial x_{i}^{k}} \frac{\partial^{\ell}}{\partial q_{j}^{\ell}}\left(\widehat{\varphi}-I_{h_{m}} \widehat{\varphi}\right)\right\|_{L^{\infty}(\Omega \times D)}<\frac{1}{m}, \quad m=1,2, \ldots
$$

Hence, passing to the limit $m \rightarrow \infty$, we deduce (3.21). 
REMARK 3.5. Hypotheses (3.14), (3.15), and (3.16) are standard assumptions for inf-sup stable $\underset{\sim}{H}(\Omega) \times L_{0}^{2}(\Omega)$-conforming discretizations of the Stokes problem. Numerous examples of finite element spaces that satisfy these properties are given in the monographs of Brezzi and Fortin [10] and Girault and Raviart [23], when $\Omega$ is a polygon in $\mathbb{R}^{2}$ or a polyhedron in $\mathbb{R}^{3}$; see also Section 4.2 in the book of Ern and Guermond [20].

Any $H^{1}(\Omega)$-conforming finite element space $\widehat{X}_{h}^{x}$ is automatically a subset of $W^{1, \infty}(\Omega)$. Similarly, any $H^{1}(D)$-conforming finite element space $\widehat{X}_{h}^{q}$ is automatically a subset of $W^{1, \infty}(D)$. Given two such spaces $\widehat{X}_{h}^{x}$ and $\widehat{X}_{h}^{q}$ we then also have $\widehat{X}_{h}:=\widehat{X}_{h}^{x} \otimes \widehat{X}_{h}^{q} \subset W^{(1,1), \infty}(\Omega \times D)$ and, trivially $\widehat{X}_{h} \subset H^{1}(\Omega \times D) \subset \widehat{X}$. The requirement (3.20) follows from standard approximation properties of classical $H^{1}$-conforming finite element spaces (see, for example, inequalities (4.4.22) and (4.4.26) in Brenner and Scott [9] when the domain is a polytope, or Theorem 4.3.4 in Ciarlet [13] and the paper of Bernardi [5] in the case of a Lipschitz domain with a curved boundary).

Let $0=t_{0}<t_{1}<\cdots<t_{N-1}<t_{N}=T$ be a partitioning of the time interval $[0, T]$ into uniform time steps $\Delta t=t_{n}-t_{n-1}, n=1 \rightarrow N$.

Given initial data satisfying (3.10), we choose ${\underset{\sim}{h}}_{h}^{0} \in \underset{\sim}{V_{h}}$ and $\widehat{\psi}_{h}^{0} \in \widehat{X}_{h}$ such that

$$
\begin{aligned}
& \int_{\Omega}\left[u_{\sim}^{0} \cdot \underset{\sim}{v_{h}}+\Delta t \underset{\sim}{\nabla_{x}} \underset{\sim}{u_{h}^{0}}: \underset{\sim}{\nabla_{x}} \underset{\sim}{w_{h}}\right] \mathrm{d} x=\int_{\sim} \underset{\sim}{u^{0} \cdot \underset{\sim}{v_{h}} \mathrm{~d} x} \underset{\sim}{\forall v_{\sim}} \in \underset{\sim}{V_{h}}, \\
& \int_{\Omega \times D} M \widehat{\psi}_{h}^{0} \widehat{\varphi}_{h} \mathrm{~d} q \underset{\sim}{\mathrm{d} x}=\int_{\Omega \times D} M \widehat{\psi}^{0} \widehat{\varphi}_{h} \mathrm{~d} q \underset{\sim}{\mathrm{d} x} \quad \forall \widehat{\varphi}_{h} \in \underset{\sim}{X_{h}} .
\end{aligned}
$$

It follows from $(3.25 \mathrm{a}, \mathrm{b})$ that

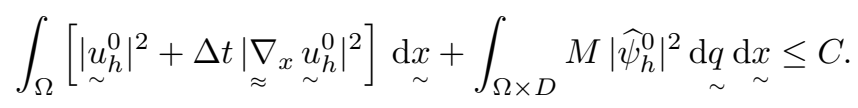

Our numerical approximation of $(\mathbf{P})$ is then defined as follows.

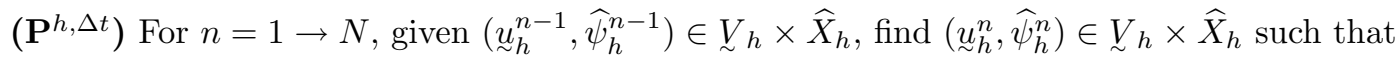

$$
\begin{aligned}
& \int_{\Omega} \frac{u_{h}^{n}-u_{\sim}^{n-1}}{\Delta t} \cdot \underset{\sim}{w_{h}} \mathrm{~d} x+\frac{1}{\sim} \int_{\Omega}\left[\left(\left(\sim_{\sim}^{n-1} \cdot \underset{\sim}{\nabla_{x}}\right) \underset{\sim}{u_{h}^{n}}\right) \cdot \underset{\sim}{w_{h}}-\left(\left(\underset{\sim}{u_{h}^{n-1}} \cdot \underset{\sim}{\nabla} x\right) \underset{\sim}{w_{h}}\right) \cdot \underset{\sim}{u_{h}^{n}}\right] \mathrm{d} x \\
& +\nu \int_{\Omega} \underset{\approx}{\nabla} \underset{\sim}{u_{h}^{n}}: \underset{\approx}{\nabla_{x}} \underset{\sim}{w_{h}} \mathrm{~d} x=-k \mu \int_{\Omega \times D} \underset{\sim}{\approx}\left(M \widehat{\psi}_{h}^{n-1}\right): \underset{\approx}{\nabla_{x}} \underset{\sim}{w_{h}} \mathrm{~d} x \quad \underset{\sim}{\forall w_{h}} \in \underset{\sim}{V_{h}},
\end{aligned}
$$

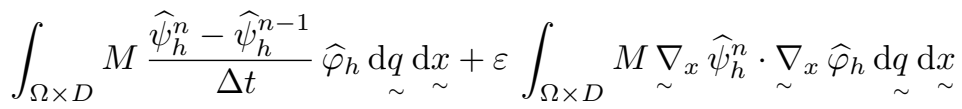

$$
\begin{aligned}
& \left.+\int_{\Omega \times D} M\left[\frac{1}{2 \lambda} \underset{\sim}{\nabla_{q}} \widehat{\psi}_{h}^{n}-\underset{\sim}{\omega}\left(u_{\sim}^{n}\right) \underset{\sim}{q}\right] \widehat{\psi}_{h}^{n}\right] \cdot \underset{\sim}{\nabla_{q}} \widehat{\varphi}_{h} \mathrm{~d} q \underset{\sim}{\sim} \mathrm{d} x \\
& +\frac{1}{2} \int_{\Omega \times D} M\left[\left({\underset{\sim}{u}}_{h}^{n} \cdot{\underset{\sim}{x}}_{x} \widehat{\psi}_{h}^{n}\right) \widehat{\varphi}_{h}-\left(\underset{\sim}{u_{h}^{n}} \cdot \underset{\sim}{\nabla_{x}} \widehat{\varphi}_{h}\right) \widehat{\psi}_{h}^{n}\right] \underset{\sim}{\mathrm{d} q \mathrm{~d} x} \underset{\sim}{\mathrm{d}}=0 \quad \forall \widehat{\varphi}_{h} \in \widehat{X}_{h} .
\end{aligned}
$$

Hence the approximations $u_{h}^{n}$ and $\widehat{\psi}_{h}^{n}$ at time level $t_{n}$ to the velocity field and the probability distribution are obtained by solving two decoupled linear equations. It is convenient to rewrite (3.27a) as

$$
b\left({\underset{\sim}{u}}_{h}^{n-1}\right)\left({\underset{\sim}{u}}_{h}^{n},{\underset{\sim}{w}}_{h}\right)=\int_{\Omega}\left[\stackrel{u}{\sim}_{h}^{n-1} \cdot{\underset{\sim}{w}}_{h}-\Delta t k \mu \underset{\sim}{C}\left(M \widehat{\psi}_{h}^{n-1}\right):{\underset{\sim}{\nabla}}_{x}\left({\underset{\sim}{w}}_{h}\right)\right] \mathrm{d} x \underset{\sim}{x} \quad \forall{\underset{\sim}{w}}_{h} \in{\underset{\sim}{V}}_{h},
$$


where for all $\underset{\sim}{v} \underset{\sim}{w_{1}},{\underset{\sim}{w}}_{2} \in \underset{\sim}{H^{1}}(\Omega)$

$$
\begin{aligned}
b(\underset{\sim}{v})\left({\underset{\sim}{1}}_{1},{\underset{\sim}{w}}_{2}\right):= & \int_{\Omega} \underset{\sim}{w_{1}} \cdot{\underset{\sim}{w}}_{2} \mathrm{~d} x+\Delta t \nu \int_{\Omega} \underset{\sim}{\nabla_{x}}{\underset{\sim}{w}}_{1}:{\underset{\sim}{\nabla}}_{x} \underset{\sim}{w_{2}} \mathrm{~d} x \\
& +\frac{\Delta t}{2} \int_{\Omega}\left[\left(\left(\underset{\sim}{v} \cdot \nabla_{x}\right) \underset{\sim}{w_{1}}\right) \cdot{\underset{\sim}{w}}_{2}-\left(\left(\underset{\sim}{v} \cdot \nabla_{x}\right) \underset{\sim}{w_{2}}\right) \cdot{\underset{\sim}{1}}_{1}\right] \mathrm{d} x .
\end{aligned}
$$

Hence it follows from $(3.29)$ that $b(\underset{\sim}{v})(\cdot, \cdot)$ is a continuous and coercive bilinear functional on $\underset{\sim}{H}(\Omega) \times$ $H^{1}(\Omega)$. On recalling (3.7), we have the right-hand side of (3.28) is a bounded linear functional on $\underset{\sim}{\sim}{ }^{1}(\Omega)$. Hence the Lax-Milgram theorem yields the existence of a unique solution $\underset{\sim}{u} \in{\underset{\sim}{V}}_{h}$ to (3.28).

It is also convenient to rewrite $(3.27 \mathrm{~b})$ as

$$
a\left(\underline{u}_{h}^{n}\right)\left(\widehat{\psi}_{h}^{n}, \widehat{\varphi}_{h}\right)=\int_{\Omega \times D} M \widehat{\psi}_{h}^{n-1} \widehat{\varphi}_{h} \mathrm{~d} \underset{\sim}{q} \mathrm{~d} x \quad \forall \widehat{\varphi}_{h} \in \widehat{X}_{h}
$$

where, for all $\widehat{\varphi}_{1}, \widehat{\varphi}_{2} \in \widehat{X}$ and $v_{h} \in \underset{\sim}{V}$,

$$
\begin{aligned}
& a\left(v_{\sim}\right)\left(\widehat{\varphi}_{1}, \widehat{\varphi}_{2}\right):=\int_{\Omega \times D} M\left(\widehat{\varphi}_{1} \widehat{\varphi}_{2}+\Delta t \varepsilon \nabla_{x} \widehat{\varphi}_{1} \cdot \nabla_{x} \widehat{\varphi}_{2}\right) \mathrm{d} \underset{\sim}{q} \mathrm{~d} x \\
& +\frac{\Delta t}{2} \int_{\Omega \times D} M\left[\left({\underset{\sim}{v} h}_{h} \cdot{\underset{\sim}{\nabla}}_{x} \widehat{\varphi}_{1}\right) \widehat{\varphi}_{2}-\left(\underset{\sim}{v_{h}} \cdot{\underset{\sim}{\nabla}}_{x} \widehat{\varphi}_{2}\right) \widehat{\varphi}_{1}\right] \mathrm{d} \underset{\sim}{q} \mathrm{~d} \underset{\sim}{x} \\
& +\Delta t \int_{\Omega \times D} M\left[\frac{1}{2 \lambda} \nabla_{q} \widehat{\varphi}_{1}-\left[\underset{\sim}{\omega}\left(v_{h}\right) \underset{\sim}{q}\right] \widehat{\varphi}_{1}\right] \cdot \nabla_{q} \widehat{\varphi}_{2} \mathrm{~d} \underset{\sim}{q} \mathrm{~d} x .
\end{aligned}
$$

In addition, it is easily deduced that $a\left(v_{\sim}\right)(\cdot, \cdot)$ is a continuous nonsymmetric bilinear functional on $\widehat{X} \times \widehat{X}$, and, for each fixed $\widehat{\psi}_{h}^{n-1} \in \widehat{X}_{h}$, the right-hand side of (3.30) defines a linear functional on $\widehat{X}$. Moreover, on noting (3.9), we see that

$$
a\left(\underset{\sim}{v_{h}}\right)(\widehat{\varphi}, \widehat{\varphi})=\int_{\Omega \times D} M\left[|\widehat{\varphi}|^{2}+\Delta t \varepsilon\left|\underset{\sim}{\nabla_{x}} \widehat{\varphi}\right|^{2}+\frac{\Delta t}{2 \lambda}\left|{\underset{\sim}{q}}_{q} \widehat{\varphi}\right|^{2}\right] \underset{\sim}{\mathrm{d} q \mathrm{~d} x} \quad \forall \widehat{\sim} \in \widehat{X} .
$$

Hence $a\left({\underset{\sim}{v}}_{h}\right)(\cdot, \cdot)$ is coercive on $\widehat{X} \times \widehat{X}$, and so the Lax-Milgram theorem yields the existence of a unique solution $\widehat{\psi}_{h}^{n} \in \widehat{X}_{h}$ to $(3.30)$.

On choosing ${\underset{\sim}{w}}_{h} \equiv{\underset{\sim}{u}}_{h}^{n}$ in (3.28) and noting (3.7) yields that

$$
\begin{aligned}
& \frac{1}{2} \int_{\Omega}\left[\left|{\underset{u}{h}}_{h}^{n}\right|^{2}+\left|{\underset{u}{u}}_{h}^{n}-{\underset{\sim}{u}}_{h}^{n-1}\right|^{2}-\left|{\underset{\sim}{u}}_{h}^{n-1}\right|^{2}\right] \mathrm{d} \underset{\sim}{x}+\Delta t \nu \int_{\Omega}\left|\underset{\approx}{\nabla} \underset{\sim}{u_{h}^{n}}\right|^{2} \mathrm{~d} \underset{\sim}{x} \\
& =-\Delta t k \mu \int_{\Omega} \underset{\approx}{C}\left(M \widehat{\psi}_{h}^{n-1}\right): \underset{\approx}{\nabla_{x}} \underset{h}{n} \mathrm{~d} \underset{\sim}{x} \leq C \Delta t \int_{\Omega \times D} M\left|\widehat{\psi}_{h}^{n-1}\right|^{2} \mathrm{~d} q \underset{\sim}{\mathrm{d}} \underset{\sim}{x} .
\end{aligned}
$$

On choosing $\underset{\sim}{w_{h}} \equiv{\underset{\sim}{Q}}_{h}\left[\underset{\sim}{S}\left(\frac{u_{h}^{n}-u_{h}^{n-1}}{\Delta t}\right)\right] \in \underset{\sim}{V}$ in (3.28) yields, on noting (3.18), (3.2), (3.19) and 
Sobolev embedding, that

$$
\begin{aligned}
& \left\|\underset{\sim}{S}\left(\frac{\underset{\sim}{u_{h}^{n}-u_{\sim}^{n-1}}}{\Delta t}\right)\right\|_{H^{1}(\Omega)}^{2}=\int_{\Omega}\left(\frac{\underset{\sim}{u_{h}^{n}-u_{h}^{n-1}}}{\Delta t}\right) \cdot \underset{\sim}{Q_{h}}\left[\underset{\sim}{S}\left(\frac{\underset{\sim}{u_{h}^{n}-u_{\sim}^{n-1}}}{\Delta t}\right)\right] \mathrm{d} \underset{\sim}{\sim} \\
& =-\nu \int_{\Omega} \underset{\sim}{\underset{\sim}{x_{x}}} \underset{\sim}{u_{h}^{n}}: \underset{\sim}{\nabla_{x}}\left[\underset{\sim}{Q_{h}}\left[\underset{\sim}{S}\left(\frac{u_{h}^{n}-u_{\sim}^{n-1}}{\Delta t}\right)\right]\right] \mathrm{d} \underset{\sim}{\mathrm{d}} \\
& -k \mu \int_{\Omega} \underset{\approx}{\approx}\left(M \widehat{\psi}_{h}^{n-1}\right): \underset{\approx}{\nabla_{x}}\left[\underset{\sim}{Q_{h}}\left[\underset{\sim}{S}\left(\frac{\underset{\sim}{u_{h}^{n}-u_{h}^{n-1}}}{\Delta t}\right)\right]\right] \mathrm{d} \underset{\sim}{\sim}
\end{aligned}
$$

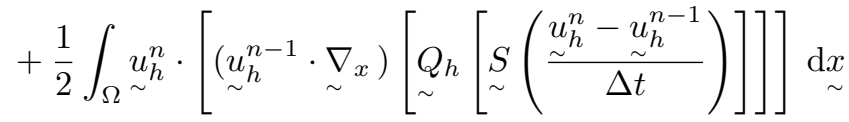

$$
\begin{aligned}
& -\frac{1}{2} \int_{\Omega}\left[\underset{\sim}{Q_{h}}\left[\underset{\sim}{S}\left(\frac{\underset{\sim}{u_{h}^{n}-u_{h}^{n-1}}}{\Delta t}\right)\right]\right] \cdot\left[\left(\underset{\sim}{\sim_{h}^{n-1}} \cdot \underset{\sim}{\nabla} x\right) \underset{\sim}{\sim_{h}^{n}}\right] \underset{\sim}{\mathrm{d} x} \\
& \leq C\left[\left\|\underset{\approx}{\|}\left(M \widehat{\psi}_{h}^{n-1}\right)\right\|_{L^{2}(\Omega)}^{2}+\left\|\underset{\approx}{\nabla_{x}} \underset{\sim}{u_{h}^{n}}\right\|_{L^{2}(\Omega)}^{2}+\left\|\left|\underset{\sim}{u_{h}^{n-1} \mid}\right| \underset{\sim}{u_{h}^{n} \mid}\right\|_{L^{2}(\Omega)}^{2}\right] \\
& +C\left\|\left|\underset{\sim}{u_{h}^{n-1}}\right|\left|\underset{\approx}{\nabla_{x}} \underset{\sim}{u_{h}^{n}}\right|\right\|_{L^{1+\delta}(\Omega)}^{2}
\end{aligned}
$$

for any $\delta>0$ if $d=2$ and for $\delta=\frac{1}{5}$ if $d=3$. Applying the Cauchy-Schwarz and the algebraicgeometric mean inequalities, in conjunction with the Gagliardo-Nirenberg inequality yields that

$$
\begin{aligned}
\left\|\left|u_{h}^{n-1}\right|\left|{\underset{\sim}{u}}_{h}^{n}\right|\right\|_{L^{2}(\Omega)}^{2} & \leq\left\|u_{\sim}^{n-1}\right\|_{L^{4}(\Omega)}^{2}\left\|u_{\sim}^{n}\right\|_{L^{4}(\Omega)}^{2} \leq \frac{1}{2} \sum_{m=n-1}^{n}\left\|u_{h}^{m}\right\|_{L^{4}(\Omega)}^{4} \\
& \leq C \sum_{m=n-1}^{n}\left[\left\|u_{\sim}^{m}\right\|_{L^{2}(\Omega)}^{4-d}\left\|\nabla_{\approx}{\underset{\sim}{u}}_{h}^{m}\right\|_{L^{2}(\Omega)}^{d}\right] .
\end{aligned}
$$

Similarly, we have for any $\delta \in(0,1)$, if $d=2$, that

$$
\begin{aligned}
& \left\|\left|\underset{\sim}{u_{h}^{n-1}}\right| \mid \underset{\approx}{\nabla_{x}} \underset{\sim}{u_{h}^{n} \mid}\right\|_{L^{1+\delta}(\Omega)}^{2} \leq\left\|\underset{\sim}{u_{h}^{n-1}}\right\|_{L^{\frac{2(1+\delta)}{1-\delta}}}^{2}\left\|\underset{\approx}{\|} \underset{\sim}{\underbrace{}_{\sim}}{\underset{\sim}{n}}_{h}^{n}\right\|_{L^{2}(\Omega)}^{2} \\
& \leq C\left\|{\underset{\sim}{u}}_{h}^{n-1}\right\|_{L^{2}(\Omega)}^{\frac{2(1-\delta)}{1+\delta}} \sum_{m=n-1}^{n}\left\|\nabla_{\approx}{\underset{\sim}{u}}_{h}^{m}\right\|_{L^{2}(\Omega)}^{\frac{2(1+3 \delta)}{1+\delta}} ;
\end{aligned}
$$

and if $d=3,\left(\delta=\frac{1}{5}\right)$, that

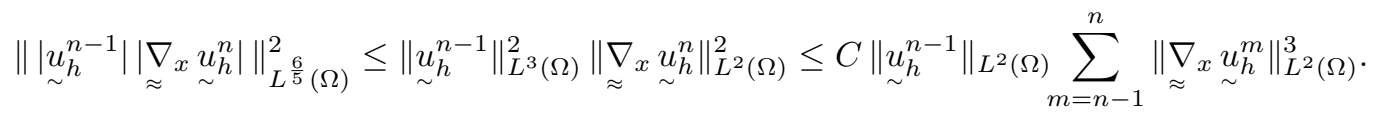

On choosing $\widehat{\varphi}_{h}=\widehat{\psi}_{h}^{n}$ in (3.30) yields that

$$
\begin{array}{r}
\int_{\Omega \times D} M\left[\left|\widehat{\psi}_{h}^{n}\right|^{2}+\left|\widehat{\psi}_{h}^{n}-\widehat{\psi}_{h}^{n-1}\right|^{2}\right] \mathrm{d} \underset{\sim}{q} \mathrm{~d} \underset{\sim}{x}+2 \Delta t \varepsilon \int_{\Omega \times D} M\left|\nabla_{x} \widehat{\psi}_{h}^{n}\right|^{2} \mathrm{~d} \underset{\sim}{q} \mathrm{~d} \underset{\sim}{\lambda} \\
+\frac{\Delta t}{\lambda} \int_{\Omega \times D} M\left|\nabla_{q} \widehat{\psi}_{h}^{n}\right|^{2} \mathrm{~d} \underset{\sim}{q} \mathrm{~d} \underset{\sim}{x}=\int_{\Omega \times D} M\left|\widehat{\psi}_{h}^{n-1}\right|^{2} \mathrm{~d} \underset{\sim}{q} \mathrm{~d} \underset{\sim}{x} ;
\end{array}
$$

where we have noted (3.9) and the simple identity

$$
2\left(s_{1}-s_{2}\right) s_{1}=s_{1}^{2}+\left(s_{1}-s_{2}\right)^{2}-s_{2}^{2} \quad \forall s_{1}, s_{2} \in \mathbb{R} .
$$


Summing (3.37) and (3.33) from $n=1 \rightarrow m$, with $1 \leq m \leq N$, and noting (3.26) and (3.7) yields that

$$
\begin{aligned}
& \max _{n=0 \rightarrow N}\left[\int_{\Omega \times D} M\left|\widehat{\psi}_{h}^{n}\right|^{2} \mathrm{~d} \underset{\sim}{q} \mathrm{~d} x\right]+\frac{1}{\lambda} \sum_{n=1}^{N} \Delta t \int_{\Omega \times D} M\left|\nabla_{q} \widehat{\psi}_{h}^{n}\right|^{2} \mathrm{~d} \underset{\sim}{q} \mathrm{~d} x \\
& +\varepsilon \sum_{n=1}^{N} \Delta t \int_{\Omega \times D} M\left|\nabla_{x} \widehat{\psi}_{h}^{n}\right|^{2} \mathrm{~d} \underset{\sim}{q} \mathrm{~d} x+\sum_{n=1}^{N} \int_{\Omega \times D} M\left|\widehat{\psi}_{h}^{n}-\widehat{\psi}_{h}^{n-1}\right|^{2} \mathrm{~d} \underset{\sim}{q} \mathrm{~d} x \\
& +\max _{n=0 \rightarrow N}\left[\int_{\Omega}\left|C\left(M \widehat{\psi}_{h}^{n}\right)\right|^{2} \mathrm{~d} x\right] \\
& \leq C \int_{\Omega \times D} M\left|\widehat{\psi}_{h}^{0}\right|^{2} \mathrm{~d} \underset{\sim}{q} \mathrm{~d} x \leq C ; \\
& \max _{n=0 \rightarrow N}\left[\int_{\Omega}\left|u_{h}^{n}\right|^{2} \mathrm{~d} x\right]+\sum_{n=1}^{N} \int_{\Omega}\left|u_{h}^{n}-u_{\sim}^{n-1}\right|^{2} \mathrm{~d} x+\nu \sum_{n=1}^{N} \Delta t \int_{\Omega}\left|\nabla_{x} u_{\sim}^{n}\right|^{2} \mathrm{~d} x \\
& \leq C \int_{\Omega}\left|{\underset{\sim}{h}}_{h}^{0}\right|^{2} \mathrm{~d} x+C T \int_{\Omega \times D} M\left|\widehat{\psi}_{h}^{0}\right|^{2} \mathrm{~d} \underset{\sim}{q} \mathrm{~d} x \leq C(T) .
\end{aligned}
$$

On taking the $\frac{2}{\beta}$ power of both sides of (3.34), recall (3.12), summing from $n=1 \rightarrow N$ and noting (3.35), (3.36a,b), (3.39a,b) and (3.26) yields that

$$
\begin{aligned}
& \sum_{n=1}^{N} \Delta t\left\|\underset{\sim}{S}\left(\frac{\underset{\sim}{u_{h}^{n}-u_{\sim}^{n-1}}}{\Delta t}\right)\right\|_{H^{1}(\Omega)}^{\frac{4}{\beta}} \\
& \leq C\left[\sum_{n=1}^{N} \Delta t \underset{\approx}{\| C}\left(M \widehat{\psi}_{h}^{n}\right) \|_{L^{2}(\Omega)}^{\frac{4}{\beta}}\right]+C(T)\left[\sum_{n=1}^{N} \Delta t\left\|\underset{\approx}{\nabla_{x}} \underset{\sim}{u_{h}^{n}}\right\|_{L^{2}(\Omega)}^{2}\right]^{\frac{2}{\beta}} \\
& +C\left[\max _{n=0 \rightarrow N}\left(\int_{\Omega}|\underset{\sim}{u}|^{2} \mathrm{~d} x\right)^{\frac{4}{\beta}-1}\right]\left[\sum_{n=0}^{N} \Delta t \int_{\Omega} \mid \nabla_{\approx} \underset{\sim}{\left.u_{h}^{n}\right|^{2} \mathrm{~d} x} \underset{\sim}{\sim}\right] \\
& \leq C(T) .
\end{aligned}
$$

Now we introduce some definitions prior to passing to the limit $h, \Delta t \rightarrow 0$. Let

$$
{\underset{\sim}{u}}_{h}^{\Delta t}(\cdot, t):=\frac{t-t_{n-1}}{\Delta t} u_{h}^{n}(\cdot)+\frac{t_{n}-t}{\Delta t} u_{h}^{n-1}(\cdot), \quad t \in\left[t_{n-1}, t_{n}\right], \quad n \geq 1,
$$

and

$$
{\underset{u}{n}}_{h}^{\Delta t,+}(\cdot, t):={\underset{\sim}{u}}_{h}^{n}(\cdot), \quad{\underset{\sim}{h}}_{h}^{\Delta t,-}(\cdot, t):={\underset{\sim}{u}}_{h}^{n-1}(\cdot), \quad t \in\left(t_{n-1}, t_{n}\right], \quad n \geq 1 .
$$

We note for future reference that

$$
\stackrel{\sim}{u}_{h}^{\Delta t}-{\underset{\sim}{u}}_{h}^{\Delta t, \pm}=\left(t-t_{n}^{ \pm}\right) \frac{\partial u_{h}^{\Delta t}}{\partial t}, \quad t \in\left(t_{n-1}, t_{n}\right), \quad n \geq 1,
$$

where $t_{n}^{+}:=t_{n}$ and $t_{n}^{-}:=t_{n-1}$. Using the above notation, and introducing analogous notation for 
$\left\{\widehat{\psi}_{h}^{n}\right\}_{n=0}^{N},(3.28)$ summed for $n=1 \rightarrow N$ can be restated as:

$$
\begin{aligned}
& \int_{0}^{T} \int_{\Omega}\left[\frac{\partial u_{h}^{\Delta t}}{\partial t} \cdot \underset{\sim}{w_{h}}+\nu \underset{\approx}{\nabla} x_{\sim}^{u_{h}^{\Delta t,+}}: \underset{\approx}{\nabla_{x}} \underset{\sim}{w_{h}}\right] \mathrm{d} x \underset{\sim}{ } \mathrm{d} t \\
& +\frac{1}{2} \int_{0}^{T} \int_{\Omega}\left[\left[\left(u_{\sim}^{\Delta t,-} \cdot \nabla_{x}\right) u_{h}^{\Delta t,+}\right] \cdot{\underset{\sim}{w}}_{h}-\left[\left(\sim_{h}^{\Delta t,-} \cdot \nabla_{x}\right){\underset{\sim}{w}}_{h}\right] \cdot{\underset{\sim}{u}}_{h}^{\Delta t,+}\right] \mathrm{d} x \underset{\sim}{ } \mathrm{d} t \\
& =-k \mu \int_{0}^{T} \int_{\Omega} \underset{\approx}{C}\left(M \widehat{\psi}_{h}^{\Delta t,-}\right): \underset{\approx}{\nabla}{\underset{\sim}{w}}_{h} \mathrm{~d} x \mathrm{~d} t \quad \forall w_{h} \in L^{\frac{4}{4-\beta}}\left(0, T ; V_{h}\right) .
\end{aligned}
$$

Similarly, (3.30) summed for $n=1 \rightarrow N$ can be restated as:

$$
\begin{aligned}
& \int_{0}^{T} \int_{\Omega \times D} M \frac{\widehat{\psi}_{h}^{\Delta t,+}-\widehat{\psi}_{h}^{\Delta t,-}}{\Delta t} \widehat{\varphi}_{h} \mathrm{~d} \underset{\sim}{q} \mathrm{~d} \underset{\sim}{x} \mathrm{~d} t+\varepsilon \int_{0}^{T} \int_{\Omega \times D} M \nabla_{x} \widehat{\psi}_{h}^{\Delta t,+} \cdot \nabla_{x} \widehat{\varphi}_{h} \mathrm{~d} \underset{\sim}{q} \mathrm{~d} x \underset{\sim}{ } \mathrm{d} t \\
& +\frac{1}{2} \int_{0}^{T} \int_{\Omega \times D} M\left[\left(\sim_{h}^{\Delta t,+} \cdot \nabla_{x} \widehat{\psi}_{h}^{\Delta t,+}\right) \widehat{\varphi}_{h}-\left({\underset{\sim}{u}}_{h}^{\Delta t,+} \cdot \nabla_{x} \widehat{\varphi}_{h}\right) \widehat{\psi}_{h}^{\Delta t,+}\right] \mathrm{d} \underset{\sim}{q} \mathrm{~d} x \underset{\sim}{x} \mathrm{~d} t
\end{aligned}
$$

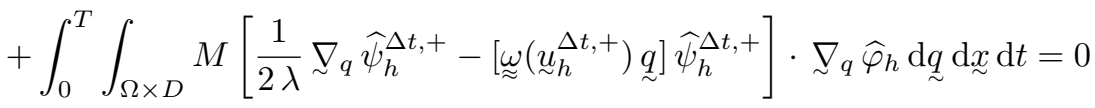

$$
\begin{aligned}
& \forall \widehat{\varphi}_{h} \in L^{2}\left(0, T ; \widehat{X}_{h}\right) \text {. }
\end{aligned}
$$

We have from (3.39a) that

$$
\begin{aligned}
& \sup _{t \in(0, T)}\left[\int_{\Omega \times D} M\left|\widehat{\psi}_{h}^{\Delta t(, \pm)}\right|^{2} \mathrm{~d} \underset{\sim}{q} \mathrm{~d} \underset{\sim}{x}\right]+\frac{1}{\lambda} \int_{0}^{T} \int_{\Omega \times D} M\left|\nabla_{q} \widehat{\psi}_{h}^{\Delta t,+}\right|^{2} \mathrm{~d} \underset{\sim}{q} \mathrm{~d} x \underset{\sim}{\mathrm{d}} t \\
& +\varepsilon \int_{0}^{T} \int_{\Omega \times D} M\left|\nabla_{x} \widehat{\psi}_{h}^{\Delta t,+}\right|^{2} \mathrm{~d} \underset{\sim}{q} \mathrm{~d} \underset{\sim}{x} \mathrm{~d} t+\sup _{t \in(0, T)}\left[\int_{\Omega}\left|C \underset{\sim}{ }\left(\widehat{\psi}_{h}^{\Delta t(, \pm)}\right)\right|^{2} \mathrm{~d} x\right] \\
& +\int_{0}^{T}\left[\int_{\Omega \times D} M \frac{\left|\widehat{\psi}_{h}^{\Delta t,+}-\widehat{\psi}_{h}^{\Delta t,-}\right|^{2}}{\Delta t} \mathrm{~d} \underset{\sim}{q} \mathrm{~d} x\right] \mathrm{d} t \leq C(T) .
\end{aligned}
$$

In the above and throughout, the notation $\widehat{\psi}_{h}^{\Delta t(, \pm)}$ means $\widehat{\psi}_{h}^{\Delta t}$ with or without the superscripts \pm . Similarly, we have from (3.39b), and (3.40) that

$$
\begin{aligned}
& \sup _{t \in(0, T)}\left[\int_{\Omega}\left|\underset{\sim}{u_{h}^{\Delta t(, \pm)}}\right|^{2} \mathrm{~d} x\right]+\int_{0}^{T} \int_{\Omega} \frac{\underset{\sim}{\left|u_{h}^{\Delta t,+}-{\underset{\sim}{h}}_{h}^{\Delta t,-}\right|^{2}}}{\Delta t} \mathrm{~d} \underset{\sim}{x} \mathrm{~d} t
\end{aligned}
$$

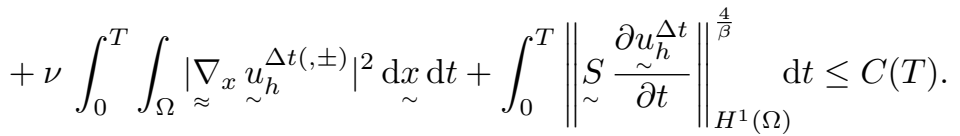

We are now in a position to prove the following convergence result.

THEOREM 3.6. There exists a subsequence of $\left\{\left(u_{h}^{\Delta t}, \widehat{\psi}_{h}^{\Delta t}\right)\right\}_{h>0, \Delta t>0}$, and functions $\underset{\sim}{u} \in L^{\infty}(0, T$; $\left.\stackrel{\sim}{L}^{2}(\Omega)\right) \cap L^{2}(0, T ; \underset{\sim}{V}) \cap W^{1, \frac{4}{\beta}}\left(0, T ; \sim^{\prime}\right)$ and $\widehat{\psi} \in L^{2}(0, T ; \widehat{X})$ with $M^{\frac{1}{2}} \widehat{\psi} \in L^{\infty}\left(0, T ; L^{2}(\widetilde{\Omega} \times D)\right)$, such that, as $h, \Delta t \rightarrow 0$,

$$
\begin{aligned}
& M^{\frac{1}{2}} \widehat{\psi}_{h}^{\Delta t(, \pm)} \rightarrow M^{\frac{1}{2}} \widehat{\psi} \quad \text { weak }{ }^{*} \text { in } L^{\infty}\left(0, T ; L^{2}(\Omega \times D)\right), \\
& M^{\frac{1}{2}} \nabla_{q} \widehat{\psi}_{h}^{\Delta t,+} \rightarrow M^{\frac{1}{2}} \nabla_{q} \widehat{\psi} \quad \text { weakly in } L^{2}\left(0, T ; L^{2}(\Omega \times D)\right) \text {, } \\
& M^{\frac{1}{2}} \underset{\sim}{\sim} \widehat{\sim}_{x} \widehat{\psi}_{h}^{\Delta t,+} \rightarrow M^{\frac{1}{2}} \underset{\sim}{\nabla}{ }_{x} \widehat{\psi} \quad \text { weakly in } L^{2}\left(0, T ; \underset{\sim}{L^{2}}(\Omega \times D)\right) \text {, } \\
& \underset{\approx}{C}\left(M \widehat{\psi}_{h}^{\Delta t(, \pm)}\right) \rightarrow \underset{\approx}{C}(M \widehat{\psi}) \quad \text { weak* in } L^{\infty}\left(0, T ; \underset{\approx}{\left.L^{2}(\Omega)\right)}\right. \text {; }
\end{aligned}
$$


and

$$
\begin{aligned}
& \underset{\sim}{u_{h}^{\Delta t(, \pm)} \rightarrow u} \underset{\sim}{u} \quad \text { weak }^{*} \text { in } L^{\infty}\left(0, T ; \underset{\sim}{\left.L^{2}(\Omega)\right),}\right. \\
& \underset{\sim}{u_{h}^{\Delta t(, \pm)} \rightarrow u} \quad \underset{\sim}{\sim} \quad \text { weakly in } L^{2}(0, T ; \underset{\sim}{V}) \text {, } \\
& \underset{\sim}{S} \frac{\partial u_{h}^{\Delta t}}{\partial t} \rightarrow \underset{\sim}{S} \underset{\sim}{\partial t} \quad \text { weakly in } L^{\frac{4}{\beta}}(0, T ; \underset{\sim}{V}) \text {, } \\
& u_{\sim}^{\Delta t(, \pm)} \rightarrow \underset{\sim}{u} \quad \text { strongly in } L^{2}\left(0, T ; \underset{\sim}{L^{r}}(\Omega),\right.
\end{aligned}
$$

where $\beta$ is defined by (3.12) and $r \in[1, \infty)$ if $d=2$ and $r \in[1,6)$ if $d=3$. Furthermore, the pair $(\underset{\sim}{u}, \widehat{\psi})$ is a weak solution of the problem $(\mathbf{P})$ defined by $\left(\mathbf{P}_{\mathbf{w}}\right)$.

Proof. The result (3.47a) follows immediately from the bounds on the first and fifth terms on the left-hand side of (3.45), on noting the notation (3.41a,b).

It follows immediately from the bound on the second term on the left-hand side of (3.45) that (3.47b) holds for some limit $\underset{\sim}{g} \in L^{2}\left(0, T ; \underset{\sim}{L^{2}}(\Omega \times D)\right)$, which we need to identify. However for any $\underset{\sim}{\eta} \in L^{2}(0, T ; \underset{\sim}{\infty}(\Omega \times D))$, it follows from $(2.7)$ and the compact support of $\underset{\sim}{\eta}$ on $D$ that $\left[\nabla_{q} \cdot\left(M^{\frac{1}{2}} \eta\right)\right] / M^{\frac{1}{2}} \in L^{2}\left(0, T ; L^{2}(\Omega \times D)\right)$ and hence the above convergence implies, on noting (3.47a), that

$$
\begin{aligned}
& \int_{0}^{T} \int_{\Omega \times D} \underset{\sim}{g} \cdot \underset{\sim}{\eta} \mathrm{d} \underset{\sim}{q} \mathrm{~d} \underset{\sim}{\mathrm{d}} \mathrm{d} t \leftarrow-\int_{0}^{T} \int_{\Omega \times D} M^{\frac{1}{2}} \widehat{\psi}_{h}^{\Delta t,+} \frac{\nabla_{q} \cdot\left(M^{\frac{1}{2}} \underset{\sim}{\eta}\right)}{M^{\frac{1}{2}}} \mathrm{~d} \underset{\sim}{q} \mathrm{~d} \underset{\sim}{\mathrm{d}} \mathrm{d} t \\
& \rightarrow-\int_{0}^{T} \int_{\Omega \times D} M^{\frac{1}{2}} \widehat{\psi} \frac{\underset{\nabla_{q}}{\underset{ }{*}\left(M^{\frac{1}{2}} \underset{\sim}{\eta}\right)}}{M^{\frac{1}{2}}} \mathrm{~d} \underset{\sim}{q} \mathrm{~d} \underset{\sim}{x} \mathrm{~d} t
\end{aligned}
$$

as $h, \Delta t \rightarrow 0$. Hence the desired result (3.47b) follows from (3.49) on noting the denseness of $C_{0}^{\infty}(\Omega \times D)$ in $L^{2}(\Omega \times D)$. A similar argument also proves (3.47c). The desired result (3.47d) follows immediately from (3.47a), and (2.3a).

The results $(3.48 \mathrm{a}-\mathrm{c})$ follow immediately from the bounds (3.46) on noting (3.15) and the denseness of $\bigcup_{h>0} R_{h}$ in $L_{0}^{2}(\Omega)$. The strong convergence result (3.48d) for $\underset{\sim}{\sim} \underset{h}{\Delta t}$ follows immediately from $(3.48 \mathrm{a}-\mathrm{c}),(3.3)$ and a standard compactness result, on noting that $\underset{\sim}{\mathrm{V}} \underset{\sim}{H_{0}^{1}}(\Omega)$ is compactly embedded in ${\underset{\sim}{r}}^{r}(\Omega)$ for the stated values of $r$. We now prove (3.48d) for $u_{h}^{\Delta t, \pm}$. First we obtain from the bound on the second term on the left-hand side of (3.46) and (3.42) that

$$
\left\|u_{h}^{\Delta t}-{\underset{u}{u}}_{h}^{\Delta t, \pm}\right\|_{L^{2}\left(0, T, L^{2}(\Omega)\right)}^{2} \leq C \Delta t .
$$

Second, we note from Sobolev embedding that, for all $\eta \in L^{2}\left(0, T ; H^{1}(\Omega)\right)$,

$$
\|\eta\|_{L^{2}\left(0, T ; L^{r}(\Omega)\right)} \leq\|\eta\|_{L^{2}\left(0, T ; L^{2}(\Omega)\right)}^{\zeta}\|\eta\|_{L^{2}\left(0, T ; L^{s}(\Omega)\right)}^{1-\zeta} \leq C\|\eta\|_{L^{2}\left(0, T ; L^{2}(\Omega)\right)}^{\zeta}\|\eta\|_{L^{2}\left(0, T ; H^{1}(\Omega)\right)}^{1-\zeta}
$$

for all $r \in[2, s)$, with any $s \in(2, \infty)$ if $d=2$ or any $s \in(2,6]$ if $d=3$, and $\zeta=[2(s-r)] /[r(s-2)] \in$ $(0,1]$. Hence, combining (3.50), (3.51) and (3.48d) for $u_{h}^{\Delta t}$ yields (3.48d) for $u_{h}^{\Delta t, \pm}$.

It remains to prove that the pair $(u, \widehat{\psi})$ is a weak solution of the problem $(\mathbf{P})$ defined by $\left(\mathbf{P}_{\mathbf{w}}\right)$. We note that for all $\underset{\sim}{v} \in L^{\infty}\left(0, T ; \underset{\sim}{L^{2}}(\Omega)\right) \cap L^{2}(0, T ; \underset{\sim}{V})$ and $\underset{\sim}{w} \in L^{2}(0, T ; \underset{\sim}{V})$ that

$$
\int_{0}^{T} \int_{\Omega}\left[\left[\left(\underset{\sim}{v} \cdot \underset{\sim}{\nabla_{x}}\right) \underset{\sim}{v}\right] \cdot \underset{\sim}{w}+\left[\left(\underset{\sim}{v} \underset{\sim}{\nabla_{x}}\right) \underset{\sim}{w}\right] \cdot \underset{\sim}{v}\right] \underset{\sim}{\mathrm{d} x} \mathrm{~d} t=\int_{0}^{T} \int_{\Omega} \underset{\sim}{\nabla_{x}} \cdot[\underset{\sim}{v} \underset{\sim}{w} \underset{\sim}{v}] \mathrm{d} x \mathrm{\sim} \mathrm{d} t=0 .
$$

It then follows from $(3.17),(3.45),(3.46),(3.48 \mathrm{a}-\mathrm{d}),(3.47 \mathrm{~d}),(3.2)$ and $(3.52)$ that we may pass to the limit, $h, \Delta t \rightarrow 0$, in $(3.43)$ to obtain that $\underset{\sim}{u} \in L^{\infty}\left(0, T ; L_{\sim}^{2}(\Omega)\right) \cap L^{2}(0, T ; V) \cap W^{1, \frac{4}{\beta}}\left(0, T ; V_{\sim}^{\prime}\right)$ and 
$\underset{\approx}{C}(M \widehat{\psi}) \in L^{\infty}\left(0, T ; \underset{\sim}{L^{2}}(\Omega)\right)$ satisfy (3.11a). It also follows from (3.25a), (3.17), (3.46) and (3.48d) that $\underset{\sim}{u}(\cdot, 0)=\sim_{\sim}^{0}(\cdot)$ in the required sense.

As we have no control of the time derivative $\widehat{\psi}_{h}^{\Delta t}$, in order to pass to the $h, \Delta t \rightarrow 0$ limit in (3.44) this derivative has to be transferred to the test function. We have for any fixed $\widehat{\varphi}_{h} \in$ $C_{0}^{\infty}\left((-T, T) ; \widehat{X}_{h}\right)$ and for $\Delta t$ sufficiently small that

$$
\begin{aligned}
& \int_{0}^{T} \int_{\Omega \times D} M \frac{\widehat{\psi}_{h}^{\Delta t,+}(\underset{\sim}{x}, \underset{\sim}{q}, t)-\widehat{\psi}_{h}^{\Delta t,-}(\underset{\sim}{x}, \underset{\sim}{q}, t)}{\Delta t} \widehat{\varphi}_{h}(\underset{\sim}{x} \underset{\sim}{q, t}) \mathrm{d} \underset{\sim}{q} \mathrm{~d} x \mathrm{~d} t \\
& =-\int_{0}^{T} \int_{\Omega \times D} M \widehat{\psi}_{h}^{\Delta t,-}(\underset{\sim}{x}, \underset{\sim}{q}, t) \frac{\widehat{\varphi}_{h}(\underset{\sim}{x}, \underset{\sim}{q}, t)-\widehat{\varphi}_{h}(\underset{\sim}{x}, \underset{\sim}{q}, t-\Delta t)}{\Delta t} \mathrm{~d} \underset{\sim}{q} \mathrm{~d} \underset{\sim}{x} \mathrm{~d} t \\
& -\int_{\Omega \times D} M \widehat{\psi}_{h}^{0}(\underset{\sim}{x} \underset{\sim}{q})\left(\frac{1}{\Delta t} \int_{0}^{t_{1}} \widehat{\varphi}_{h}(\underset{\sim}{x}, \underset{\sim}{q}, t-\Delta t) \mathrm{d} t\right) \mathrm{d} \underset{\sim}{q} \mathrm{~d} x .
\end{aligned}
$$

It follows for all $\widehat{\varphi}_{h} \in C_{0}^{\infty}\left((-T, T) ; \widehat{X}_{h}\right)$ and for all $\left.\underset{\sim}{x} \underset{\sim}{q}, t\right) \in \Omega \times D \times(0, T)$ that

$$
\frac{\widehat{\varphi}_{h}(\underset{\sim}{x}, \underset{\sim}{q}, t)-\widehat{\varphi}_{h}(\underset{\sim}{x}, \underset{\sim}{q}, t-\Delta t)}{\Delta t}=\frac{\partial \widehat{\varphi}_{h}}{\partial t}(\underset{\sim}{x} \underset{\sim}{q}, t)+R_{\Delta t}\left(\widehat{\varphi}_{h}\right)(\underset{\sim}{x}, \underset{\sim}{q}, t),
$$

where

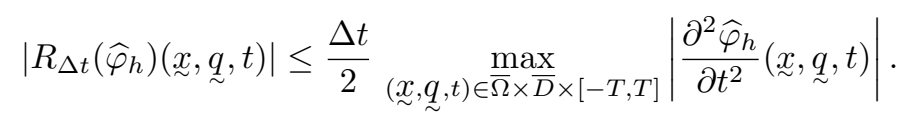

Next, we note that for all $\underset{\sim}{v} \in \underset{\sim}{H}(\Omega)$ and $\underset{\sim}{\eta} \in H^{1}\left(\Omega ; L^{2}(D ; M)\right)$ we have

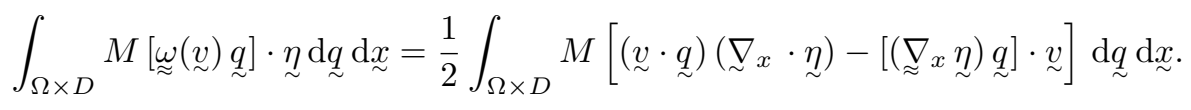

Hence, on combining (3.44) for a fixed $\widehat{\varphi}_{h} \in C_{0}^{\infty}\left((-T, T) ; \widehat{X}_{h}\right),(3.53),(3.54),(3.25 \mathrm{~b})$ and $(3.55)$ with $\underset{\sim}{\eta}=\widehat{\psi}_{h} \nabla_{q} \widehat{\varphi}_{h}$ we have for $\Delta t$ sufficiently small that

$$
\begin{aligned}
& -\int_{0}^{T} \int_{\Omega \times D} M \widehat{\psi}_{h}^{\Delta t,-}\left[\frac{\partial \widehat{\varphi}_{h}}{\partial t}+R_{\Delta t}\left(\widehat{\varphi}_{h}\right)\right] \underset{\sim}{\mathrm{d} q} \mathrm{~d} x \mathrm{~d} t
\end{aligned}
$$

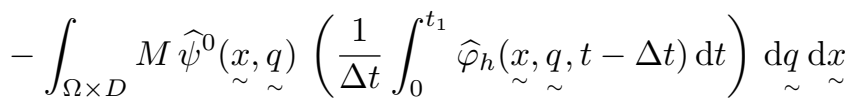

$$
\begin{aligned}
& +\int_{0}^{T} \int_{\Omega \times D} M\left[\frac{1}{2 \lambda} \underset{\sim}{\nabla} q \widehat{\psi}_{h}^{\Delta t,+} \cdot \underset{\sim}{\nabla_{q}} \widehat{\varphi}_{h}+\underset{\sim}{\varepsilon} \underset{\sim}{\nabla} \widehat{\psi}_{h}^{\Delta t,+} \cdot \underset{\sim}{\nabla_{x}} \widehat{\varphi}_{h}\right] \underset{\sim}{\mathrm{d} q} \mathrm{~d} \underset{\sim}{x} \mathrm{~d} t \\
& +\frac{1}{2} \int_{0}^{T} \int_{\Omega \times D} M\left[\left(u_{\sim}^{\Delta t,+} \cdot \underset{\sim}{\nabla} \widehat{\psi}_{h}^{\Delta t,+}\right) \widehat{\varphi}_{h}-\left(u_{\sim}^{\Delta t,+} \cdot \underset{\sim}{\nabla_{x}} \widehat{\varphi}_{h}\right) \widehat{\psi}_{h}^{\Delta t,+}\right] \underset{\sim}{\mathrm{d} q} \mathrm{~d} x \mathrm{\sim} \mathrm{d} t \\
& \left.+\frac{1}{2} \int_{0}^{T} \int_{\Omega \times D} M\left[\left[\underset{\sim}{\underset{\sim}{\nabla}}\left(\widehat{\psi}_{h} \underset{\sim}{\nabla_{q}} \widehat{\varphi}_{h}\right) \underset{\sim}{q}\right] \cdot{\underset{\sim}{u}}_{h}^{\Delta t,+}-\underset{\sim}{\sim_{h}^{\Delta t,+}} \cdot \underset{\sim}{q}\right)\left[\underset{\sim}{\nabla_{x}} \cdot\left(\widehat{\psi}_{h} \underset{\sim}{\nabla_{q}} \widehat{\varphi}_{h}\right)\right]\right] \underset{\sim}{\mathrm{d} q} \mathrm{~d} x \mathrm{\sim} d t=0 .
\end{aligned}
$$

Similarly to $(3.52)$, we note that for all $\underset{\sim}{v} \in L^{\infty}\left(0, T ; L_{\sim}^{2}(\Omega)\right) \cap L^{2}(0, T ; \underset{\sim}{V})$ and $\widehat{\varphi}_{1}, \widehat{\varphi}_{2} \in$ $L^{2}(0, T ; \widehat{X})$ that

$$
\begin{aligned}
\int_{0}^{T} \int_{\Omega \times D} M\left[\left[\underset{\sim}{v} \cdot \underset{\sim}{\nabla_{x}}\right) \widehat{\varphi}_{1}\right] \cdot \widehat{\varphi}_{2}+ & {\left.\left.\left[\underset{\sim}{(v} \underset{\sim}{\nabla_{x}}\right) \widehat{\varphi}_{2}\right] \cdot \widehat{\varphi}_{1}\right] \underset{\sim}{\mathrm{d} q} \mathrm{~d} x \mathrm{\sim} \mathrm{d} t } \\
& =\int_{0}^{T} \int_{\Omega \times D} \underset{\sim}{\nabla_{x}} \cdot\left[M\left(\varphi_{1} \cdot \varphi_{2}\right) \underset{\sim}{v]} \underset{\sim}{\mathrm{d}} \underset{\sim}{\mathrm{d}} x \mathrm{~d} t=0 .\right.
\end{aligned}
$$


Let us consider (3.56) with $h=h_{m}, m=1,2, \ldots$, where $h_{m}$ is as in $(3.21)$, and take $\widehat{\varphi}_{h}(\cdot, t)=$ $\widehat{\varphi}_{h_{m}}(\cdot, t)=I_{h_{m}} \widehat{\varphi}(\cdot, t)$ for $t \in[-T, T]$, with $\widehat{\varphi} \in C_{0}^{\infty}\left((-T, T) ; C^{\infty}(\overline{\Omega \times D})\right)$. It then follows from (3.21), (3.45), (3.46), (3.47a-c), (3.48d), (3.54) and (3.57) with $h=h_{m}$, that we may pass to the limit $h_{m} \rightarrow 0$ (on letting $m \rightarrow \infty$ ) and $\Delta t \rightarrow 0$ in (3.56) to obtain that $\widehat{\psi} \in L^{2}(0, T ; \widehat{X})$ with $M^{\frac{1}{2}} \psi \in$ $L^{\infty}\left(0, T ; L^{2}(\Omega \times D)\right)$ and $\underset{\sim}{u} \in L^{2}(0, T ; \underset{\sim}{V})$ satisfy $(3.11 \mathrm{~b})$ for any $\widehat{\varphi} \in C_{0}^{\infty}\left((-T, T) ; C^{\infty}(\overline{\Omega \times D})\right)$. Finally, noting that $C_{0}^{\infty}\left((-T, T) ; C^{\infty}(\overline{\Omega \times D})\right)$ is a dense subset of $\widehat{\mathcal{X}}$, recall (3.13), it follows that (3.11b) remains true for all $\widehat{\varphi} \in \widehat{\mathcal{X}}$. Hence we have proved, using a weak-compactness argument, the existence of a global weak solution of $(\mathbf{P}),(3.11 \mathrm{a}, \mathrm{b})$, as well as that a (sub)sequence of approximations generated by the proposed numerical method converges, in the sense of $(3.47 \mathrm{a}-\mathrm{d})$ and $(3.48 \mathrm{a}-\mathrm{d})$, to such a global weak solution.

In our forthcoming paper [4] we shall establish an analogous convergence result in the technically more involved case of a general noncorotational dumbell model for a dilute polymer. There, we shall confine ourselves to spatial discretizations based on the finite element method.

\section{REFERENCES}

[1] L. Ambrosio, Transport equation and Cauchy problem for BV vector fields, Inven. Math. 158 (2004) $227-260$.

[2] J.W. Barrett, Ch. Schwab, and E. Süli, Existence of global weak solutions for some polymeric flow models, Math. Models and Methods in Applied Sciences 15 (2005) 939-983.

[3] J.W. Barrett and E. Süli, Existence of global weak solutions to some regularized kinetic models of dilute polymers, SIAM Multiscale Modelling and Simulation 6 (2007) 506-546.

[4] J.W. Barrett and E. Süli, Finite element approximation of kinetic dilute polymer models with microscopic cut-off, In preparation, 2007.

[5] C. Bernardi, Optimal finite element interpolation on curved domains, SIAM J. Numer. Anal. 26 (1989) 12121240.

[6] A.V. Bhave, R.C. Armstrong, and R.A. Brown, Kinetic theory and rheology of dilute, nonhomogeneous polymer solutions, J. Chem. Phys. 95 (1991) 2988-3000.

[7] R. Bird, C. Curtiss, R. Armstrong, and O. Hassager, Dynamics of Polymeric Liquids, Vol 2: Kinetic Theory (John Wiley \& Sons, 1987).

[8] J. H. Bramble, J. E. Pasciak, and O. Steinbach, On the stability of the $L_{2}$ projection in $H_{0}^{1}$, Math. Comput. 71 (2002) 147-156.

[9] S.C. Brenner and L.R. Scott, The Mathematical Theory of Finite Element Methods, Second Edition (SpringerVerlag, 2002).

[10] F. Brezzi, M. Fortin, Mixed and Hybrid Finite Element Methods, Springer Series in Computational Mathematics (Springer-Verlag, 1991).

[11] C. Carstensen, Merging the Bramble-Pasciak-Steinbach and the Crouzeix-Thomée criterion for $H^{1}$-stability of the $L_{2}$-projection onto finite element spaces, Math. Comput. 71 (2002) 157-163.

[12] S. Cerrai, Second-order PDEs in finite and infinite dimension, Lecture Notes in Mathematics 1762 (SpringerVerlag, 2001).

[13] Ph. Ciarlet, The Finite Element Method for Elliptic Problems (North-Holland, 1978).

[14] P. Constantin, Nonlinear Fokker-Planck Navier-Stokes systems, Comm. Math. Sci. 3 (2005) 531-544.

[15] M. Crouzeix and V. Thomée, The stability in $L_{p}$ and $W_{p}^{1}$ of the $L_{2}$-projection onto finite element function spaces, Math. Comput. 48 (1987) 521-532.

[16] R.J. DiPerna and P.-L. Lions, Ordinary differential equations, transport theory and Sobolev spaces, Inventiones Mathematicae 98 (1989) 511-547.

[17] Q. Du, C. Liu, and P. Yu, FENE dumbbell models and its several linear and nonlinear closure approximations, SIAM Multiscale Modelling and Simulations 4 (2005) 709-731.

[18] W. E, T.J. Li, and P.-W. Zhang, Well-posedness for the dumbbell model of polymeric fluids, Commun. Math. Phys. 248 (2004) 409-427.

[19] A.W. El-Kareh and L.G. Leal, Existence of solutions for all Deborah numbers for a non-Newtonian model modified to include diffusion, J. Non-Newtonian Fluid Mech. 33 (1989) 257-287.

[20] A. Ern and J.-L. Guermond, Theory and Practice of Finite Elements (Springer-Verlag, 2004).

[21] J.-Cl. Evard and F. Jafari, Direct computation of the simultaneous Stone-Weierstrass approximation of a function and its partial derivatives in Banach spaces and combination with Hermite interpolation, J. Approx. Th. 78 (1994) 351-363.

[22] C. Foias, D.D. Holm, and E.S. Titi, The Navier-Stokes- $\alpha$ model of fluid turbulence, Physica D 152-153 (2001) 505-519.

[23] V. Girault and P.-A. Raviart, Finite Element Methods for Navier-Stokes Equations, Springer Ser. Comp. Math. 
5 (Springer-Verlag, 1986).

[24] J.G. Heywood and R. Rannacher, Finite element approximation of the nonstationary Navier-Stokes problem I: Regularity of solutions and second-order error estimates for spatial discretization, SIAM J. Numer. Anal. 19 (1982) 275-311.

[25] B. Jourdain, T. Lelièvre, and C. Le Bris, Numerical analysis of micro-macro simulations of polymeric fluid flows: a simple case, $M^{3} A S 12$ (2002) 1205-1243.

[26] B. Jourdain, T. Lelièvre, and C. Le Bris, Existence of solution for a micro-macro model of polymeric fluid: the FENE model, J. Funct. Anal. 209 (2004) 162-193.

[27] R. Keunings, A survey of computational rheology. Proc. 13th Int. Congr. on Rheology, D.M. Binding et al. (Eds), British Society of Rheology, Glasgow, Vol. 1, 7-14 (2000). Available in electronic form from: http://www .mate.tue.nl/ hulsen/cr/Keunings00.pdf

[28] A. Kufner, Weighted Sobolev Spaces, Teubner-Texte zur Mathematik (Teubner, 1980).

[29] T. Li, H. Zhang, and P.-W. Zhang, Local existence for the dumbbell model of polymeric fuids, Commun. PDE 29 (2004) 903-923.

[30] T. Li and P.-W. Zhang, Mathematical analysis of multi-scale models of complex fluids. Commun. Math. Sci. 5 (2007) $1-51$.

[31] P.-L. Lions and N. Masmoudi, Global solutions for some Oldroyd models of non-Newtonian flows, Chin. Ann. of Math. B 21 (2000) 131-146.

[32] P.-L. Lions and N. Masmoudi, Global existence of weak solutions to some micro-macro models, C. R. Math. Acad. Sci. Paris (to appear). Available from: http://www.math.nyu.edu/faculty/masmoudi/index.html

[33] L. Lorenzi and M. Bertoldi, Analytical Methods for Markov Semigroups (CRC Press, New York, 2006).

[34] A. Lozinski, C. Chauvière, J. Fang, and R.G. Owens, Fokker-Planck simulations of fast flows of melts and concentrated polymer solutions in complex geometries, J. Rheol. 47 (2003) 535-561.

[35] A. Lozinski, R.G. Owens, and J. Fang, A Fokker-Planck-based numerical method for modelling non-homogeneous flows of dilute polymeric solutions, J. Non-Newtonian Fluid Mech. 122 (2004) 273-286.

[36] N. Masmoudi, Well posedness of the FENE dumbbell model of polymeric flows. Preprint, 2007. Available from: http://www.math.nyu.edu/faculty/masmoudi/index.html

[37] L. Nachbin, Sur les algebrès denses de fonctions différentiables sur une variété, C. R. Acad. Sci. Paris. 228 (1949) 1549-1551.

[38] F. Otto and A. Tzavaras, Continuity of velocity gradients in suspensions of rod-like molecules, SFB Preprint 147, Institute of Applied Maths, Univ. Bonn, Germany (2006).

[39] H.-C. Öttinger, Stochastic Processes in Polymeric Fluids (Springer-Verlag, 1996).

[40] R. G. Owens and T. N. Phillips, Computational Rheology (Imperial College Press, 2002).

[41] M. Renardy, An existence theorem for model equations resulting from kinetic theories of polymer solutions, SIAM J. Math. Anal. 22 (1991) 313-327.

[42] J.D. Schieber, Generalized Brownian configuration field for Fokker-Planck equations including center-of-mass diffusion, J. Non-Newtonian Fluid Mech. 135 (2006) 179-181.

[43] E.M. Stein, Singular integrals and differentiability properties of functions (Princeton Univ. Press, 1970).

[44] E. Süli, Finite element approximation of high-dimensional transport-dominated diffusion problems. Foundations of Computational Mathematics 2005, Luis-Miguel Pardo, Allan Pinkus, Endre Süli, Michael Todd, editors, 343-370 (Cambridge University Press, 2006).

[45] Ch. Schwab, E. Süli, and R.-A. Todor, Sparse finite element approximation of high-dimensional transportdominated diffusion problems, M2AN: Mathematical Modelling and Numerical Analysis (Submitted for publication, 2007).

[46] R. Temam, Navier-Stokes Equations. Theory and Numerical Analysis, Third Edition, Studies in Mathematics and its Applications, 2 (North-Holland, 1984).

[47] H. Triebel, Interpolation Theory, Function Spaces, Differential Operators, Second Edition (Joh. Ambrosius Barth Publ., 1995).

[48] P. Yu, Q. Du, and C. Liu, From micro to macro dynamics via a new closure approximation to the FENE model of polymeric fluids, SIAM Multiscale Modeling and Simulations 3 (2005) 895-917. 